Article Submitted to JACS, 07/2021

\title{
A Theory-guided X-ray Absorption Spectroscopy Approach for Identifying Active Sites in Atomically Dispersed Transition Metal
}

\section{Catalysts.}

Yizhen Chen, ${ }^{1, \#}$ Rachita Rana, ${ }^{1, \#}$ Tyler Sours, ${ }^{1, \#}$ Fernando D. Vila, ${ }^{2}$ Shaohong Cao, ${ }^{3}$ Thomas Blum, ${ }^{4}$ Jiyun Hong, ${ }^{5}$ Adam S. Hoffman, ${ }^{5}$ Chia-Yu Fang, ${ }^{1}$ Zhennan Huang, ${ }^{3}$ Chunyan Shang, ${ }^{6}$ Chuanhao Wang, ${ }^{6}$ Jie Zeng, ${ }^{6}$ Miaofang Chi,${ }^{3}$ Coleman X. Kronawitter, ${ }^{1 *}$ Simon R. Bare, ${ }^{5 *}$ Bruce C. Gates ${ }^{1 *}$ Ambarish R. Kulkarni ${ }^{1 *}$

${ }^{1}$ Department of Chemical Engineering, University of California, Davis, CA 95616, USA

${ }^{2}$ Department of Physics, University of Washington, Seattle, Washington 98195, United States

${ }^{3}$ Oak Ridge National Laboratory, Oak Ridge, TN 37830, USA

${ }^{4}$ University of California Irvine, Irvine, CA 92697, USA

${ }^{5}$ Stanford Synchrotron Radiation Lightsource, SLAC National Accelerator Laboratory, Menlo Park, CA 94025, United States

${ }^{6}$ Hefei National Laboratory for Physical Sciences at the Microscale, Department of Chemical Physics, University of Science and Technology of China, Hefei, Anhui 230026, P. R. China

\# These authors contributed equally to this work

Correspondence and requests for materials should be addressed to C.X.K. (email: ckrona@ucdavis.edu), $\quad$ S.R.B. $\quad$ (email: $\quad$ srbare@ slac.stanford.edu), $\quad$ B.C.G. $\quad$ (email: bcgates@ucdavis.edu) or to A.R.K. (arkulkarni@ucdavis.edu). 


\section{ABSTRACT}

Atomically dispersed supported metal catalysts offer new properties and the benefits of maximized metal accessibility and utilization. The characterization of these materials, however, remains challenging. Using atomically-dispersed Pt supported on crystalline $\mathrm{MgO}$ (chosen for its well-defined bonding sites for $\mathrm{Pt}$ ) as a prototypical example, in this work, we demonstrate how systematic density functional theory calculations (for assessing all the potentially stable Pt sites) combined with automated EXAFS analysis can lead to unbiased identification of isolated, surfaceenveloped platinum cations as the catalytic species for $\mathrm{CO}$ oxidation. The catalyst has been characterized by atomic-resolution imaging, EXAFS, and HERFD-XANES spectroscopies; the proposed Pt site are in full agreement with experiment. This theory-guided workflow leads to rigorously determined structural models and provides a more detailed picture of the structure of the catalytically active sites than what is currently possible with conventional EXAFS analysis. As this approach is efficient and agnostic to the metal, support, and catalytic reaction, we posit that it will be of broad interest to the materials characterization and catalysis communities. 


\section{INTRODUCTION}

Atomically dispersed metals on reducible $\left(\mathrm{CeO}_{2}, \mathrm{TiO}_{2}, \mathrm{Fe}_{3} \mathrm{O}_{4}\right)$ and nonreducible $\left(\mathrm{Al}_{2} \mathrm{O}_{3}\right.$, $\mathrm{MgO}, \mathrm{ZrO}_{2}$ ) metal oxide supports have drawn wide attention recently as catalysts for a wide range of reactions including $\mathrm{CO}$ oxidation, water-gas shift, and other oxidations and reductions. ${ }^{1-8}$ These catalysts offer the advantages of maximum accessibility of the metals, which are often expensive (e.g., Pt, Pd, Ir, Rh), and catalytic properties distinct from those of the conventional supported metal clusters and nanoparticles. Like most single transition metal (TM) atoms in organometallic complexes, those on metal oxide supports are positively charged, having properties that are strongly influenced by their surroundings. ${ }^{9-12}$ These surroundings (bonding environments) are challenging to identify, because the support surfaces are inherently heterogeneous. Even when the most incisive experimental methods are used (e.g., aberration corrected scanning transmission electron microscopy (STEM), X-ray absorption spectroscopy (XAS), and probe molecule experiments with spectroscopies), the results often provide only average structural models of the dominant metal bonding environment; the possible contributions of minority catalytic species are often overlooked. ${ }^{13}$

Common research approaches involve using experimentally determined structure parameters (e.g., bond lengths and coordination numbers from extended x-ray absorption fine structure (EXAFS) spectroscopy) with complementary information characterizing local geometry and electronic structure from x-ray absorption near-edge structure (XANES) spectra. These techniques are often combined to create plausible atomistic models that are subsequently investigated using density functional theory (DFT) calculations to derive quantitative structural and mechanistic insights. ${ }^{7,14-16}$ More recent approaches have utilized machine learning techniques to gain structural information. ${ }^{17}$ 
We emphasize that, as conventionally practiced, the initial development of atomistic models relies predominantly on thorough EXAFS modeling, which is often quite demanding and time intensive for the user, especially when multiple scattering paths are considered. Today's "hands-on" EXAFS modeling approaches fall short of determining the existence of multiple metal bonding environments - a central limitation in the field. Moreover, structures inferred for the sites may not be consistent with all the complementary experimental results. As atomically dispersed catalysts continue to be reported for new reactions, there is a clear potential scientific benefit to develop computationally guided approaches for characterization of these materials, specifically for the quantitative identification of the metal site environment(s).

(a) From synchrotron measurements..

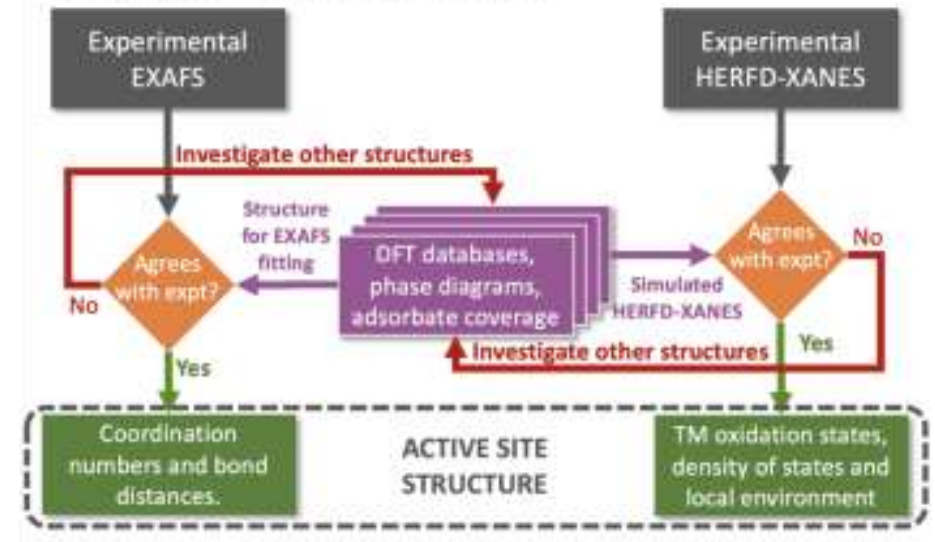

(b)

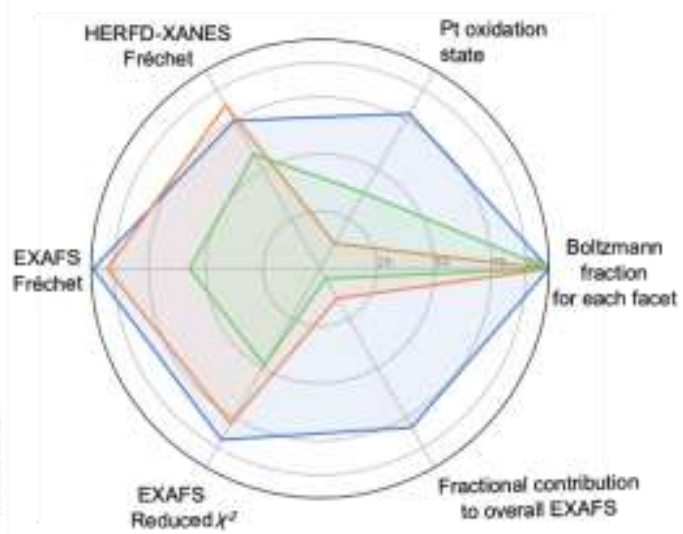

... to theory-guided interpretation

Figure 1. (a) Schematic representation of theory-guided workflow for identifying the Pt active sites stabilized within or on a MgO lattice. (b) Radar plot for the most stable [100], [100] ${ }^{\mathrm{Mg}-\mathrm{vac}}$, and [310] Pt configurations showing that the $[100]^{\mathrm{Mg}-\mathrm{vac} / \mathrm{sub} 1}$ (blue) is more consistent with our experimental observations than $[100] / \mathrm{subO} / * \mathrm{O}_{2}$ (orange) or $[310] / \mathrm{pos} /{ }^{*} \mathrm{O}_{2}$ site (green). Structure models are presented in Figure 3. 
As a point of departure from reported investigations, we now describe a theory-led workflow for characterizing atomically dispersed supported metal catalysts. Our approach combines state-of-the-art characterization techniques (high-angle annular dark field (HAADF) STEM, EXAFS spectroscopy, and high-energy resolution fluorescence detection (HERFD) XANES spectroscopy) with DFT-level theory for critical identification of the local environments for a prototypical catalyst - atomically dispersed $\mathrm{Pt}$ cations on $\mathrm{MgO}(\mathrm{Pt} / \mathrm{MgO})$. We chose $\mathrm{MgO}$ as a prototypical support because it (a) is nonreducible, (b) consists of low-atomic-number elements for excellent contrast with Pt in STEM, and (c) is available as robust, high-area crystalline materials used in industrial catalysts. ${ }^{5,10}$ These crystalline supports are expected to present limited numbers of sites for stable anchoring of isolated metal atoms, making $\mathrm{Pt} / \mathrm{MgO}$ an excellent test case to exemplify our methodology.

Figure 1a is a summary of our strategy that combines microscopy and conventional EXAFS analyses (Sections 3.1 and 3.2), large-scale DFT calculations (Section 3.3), automated DFT-based EXAFS analyses (Section 3.4), and FEFF-XANES spectroscopy (Section 3.5) to characterize $\mathrm{Pt} / \mathrm{MgO}$ and identify the structure most consistent with data obtained through all the complementary techniques. As shown throughout this report, the outcome of this approach, represented as a radar plot (Figure 1b, details in Table S1), identifies sub-surface Pt sites within $\mathrm{MgO}$ (denoted $\mathrm{Pt}^{+4} /[100]^{\mathrm{Mg}-\mathrm{vac}}$ ) to be the most populated sites for $\mathrm{Pt}$ atoms for this system. Although the approach is applied for atomically dispersed Pt/MgO (Section 3.6), we emphasize that the strategy is agnostic to the metal, the oxide support, and the reaction. Moreover, this approach can be extended to simultaneously fit multiple DFT structures to the experimental EXAFS. To our best knowledge, this approach is unique in the field and represents a significant step forward towards the goal of comprehensive, validated integration of theoretical and 
experimental methods to describe the structure and catalytic function of isolated noble metal cations stabilized by a metal oxide support.

\section{RESULTS}

\subsection{Synthesis and Structural Characterization.}

Atomically dispersed $\mathrm{Pt}$ on $\mathrm{MgO}$, prepared from ethanol-water solutions of $\mathrm{K}_{2} \mathrm{PtCl}_{4}$ and slurried $\mathrm{MgO}$ powder, was calcined at $700{ }^{\circ} \mathrm{C}$. Low Pt loadings $(\approx 0.05 \mathrm{wt} \%)$ were targeted to minimize the number of different support surface sites that the Pt atoms occupy. TEM images and $\mathrm{X}$-ray diffraction (XRD) patterns show that $\mathrm{MgO}$ was present as the cubic phase consisting of approximately 200-nm diameter crystallites; there was no XRD evidence of metallic Pt (Figure $\mathrm{S} 1)$. The calcined $\mathrm{MgO}$ was highly dehydroxylated, with only a weak $\mathrm{OH}$ band in the IR spectrum, at $3741 \mathrm{~cm}^{-1}$ (Figure S2). HAADF-STEM images show atomically dispersed Pt in the absence of clusters or nanoparticles (Figure 2, Figure S3). The bright spots in yellow circles (Figure 2a) show isolated Pt atoms along $\mathrm{Mg}$ columns in the (110) projection. ${ }^{18}$ Intensity profiles along the $\mathrm{X}-\mathrm{Y}$ line in Figure 2b (Figure 2c) confirm the isolation of the Pt atoms.
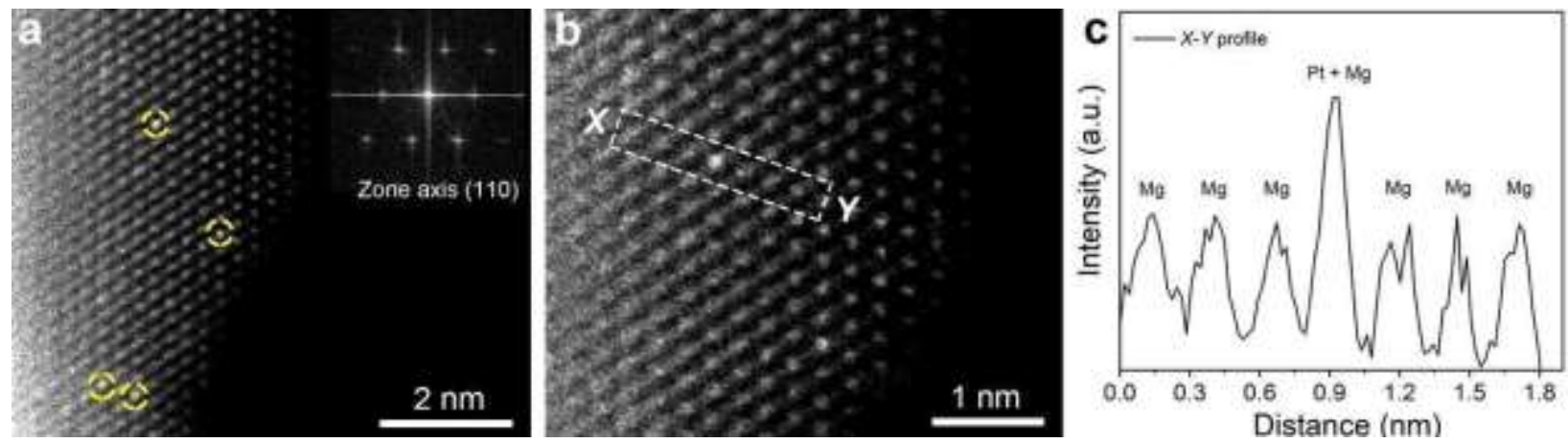

Figure 2. (a) HAADF-STEM image (along the (110) zone axis) showing that the MgO-supported Pt atoms (yellow circles) were atomically dispersed. (b) Higher magnification image showing a single isolated Pt atom, and (c) corresponding intensity profile from the $\mathrm{X}-\mathrm{Y}$ line scan in $2 \mathbf{b}$.

\subsection{Conventional EXAFS Analysis.}


$\mathrm{Pt} \mathrm{L}_{3}$-edge EXAFS spectra were modeled in Artemis ${ }^{19}$ using traditional methods. The bestfit model comprises three scattering paths: $\mathrm{Pt}-\mathrm{O}(2.05 \pm 0.01 \AA)$, with a coordination number $(\mathrm{CN})$ of $6.7 \pm 0.8$, and two Pt-Mg (3.01 \pm 0.04 and $3.15 \pm 0.04 \AA$ ), with a total CN of 11 (Figures S4-6, Table S2-4). Addition of a Pt-Pt scattering path (expected at $2.7 \AA$ for $\mathrm{Pt}^{0}$ clusters) resulted in non-meaningful results, ${ }^{20}$ confirming the atomic dispersion of Pt demonstrated by HAADFSTEM. The continuous Cauchy wavelet transform (CCWT) heatmap of the sample (Figure S7a) shows two main scattering features arising from short and long distances. ${ }^{21}$ This analysis provides evidence that the longer features at $5 \AA^{-1}$ arise from a lower-Z scattering atom than $\mathrm{Pt}$ (i.e., $\mathrm{Mg}$ ) as it does not match the features of the CCWT for Pt metal (Figure S7b). The scattering feature with a short-distance, low-Z scattering component was best-modeled as a Pt-O path. The higher- $R$ (distance) component was modeled as two $\mathrm{Pt}-\mathrm{Mg}$ paths at longer distances.

We emphasize that this conventional analysis of the modeled EXAFS data is insufficient to determine more than a single average, best-fit structure. Thus, it is difficult to define the actual bonding site. To address the possible presence of more than one significant supported Pt species and to exhaustively examine the possible Pt bonding environments, we developed an automated, DFT-based workflow for EXAFS data fitting that reduces user intervention, and thereby improves the objectivity of the data interpretation. We denote this methodology as the QuantEXAFS approach, a term that emphasizes the quantitative nature of our fitting (using hundreds of unique paths) based on structures derived from quantum chemistry calculations (i.e., DFT for the Pt/MgO system).

\subsection{A Comprehensive Library of DFT-optimized Structures.}

We used a theory-guided characterization approach to elucidate the local Pt bonding environment, that is, using DFT calculations (PBESol functional, implemented in VASP) ${ }^{22}$ to 
create a comprehensive library of all plausible $\mathrm{Pt} / \mathrm{MgO}$ structures. As summarized in Figure 3a-c, we considered three representative $\mathrm{MgO}$ facets (terrace sites: [100], Mg vacancy terrace sites: $[100]^{\mathrm{Mg}-\mathrm{vac}}$, step sites: [310]), various adsorbates $\left({ }^{*} \mathrm{O},{ }^{*} \mathrm{O}_{2}\right)$, vacancy types $\left(\mathrm{O}^{\mathrm{vac}}, \mathrm{Mg}^{\mathrm{vac}}\right)$, and subsurface Pt locations. ${ }^{23}$ The Pt sites are denoted according to the $\mathrm{MgO}$ facet, the Pt atom location, and any adsorbates or vacancies. For example, $[100]^{\mathrm{Mg}-v a c} / \mathrm{sub} 1$ refers to a 100 facet with the $\mathrm{Pt}$ atom located in the first sub-surface site next to an Mg vacancy. Furthermore, we use three colors

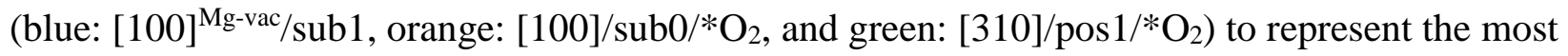
stable Pt site on each facet. These structures spanning a wide range of local Pt environments were used to construct the temperature-dependent phase diagram (using pMuTT, harmonic approximation for vibrational entropy) for each site. ${ }^{24} \mathrm{We}$ chose to analyze each facet separately as the mechanism of Mg-vacancy formation (and therefore, $\mathrm{Mg}$ chemical potential $\left(\mu_{\mathrm{Mg}}\right)$ ) during the high-temperature calcination is not known.

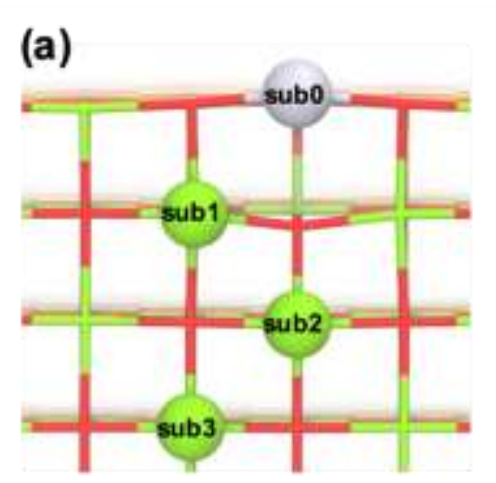

(d)
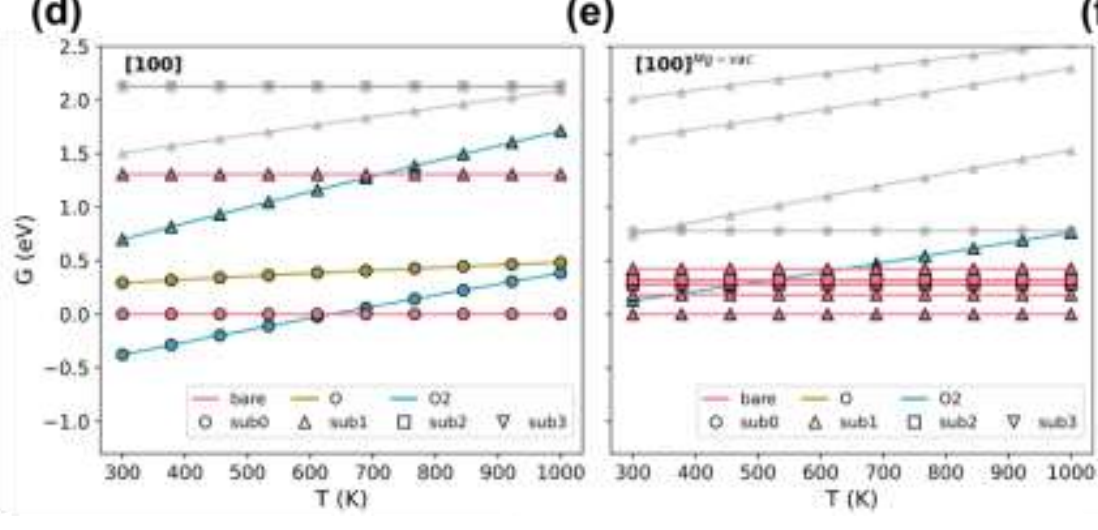

(c)

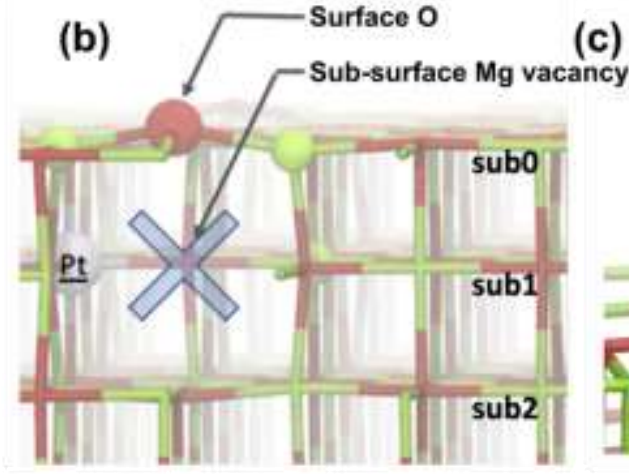

(f)

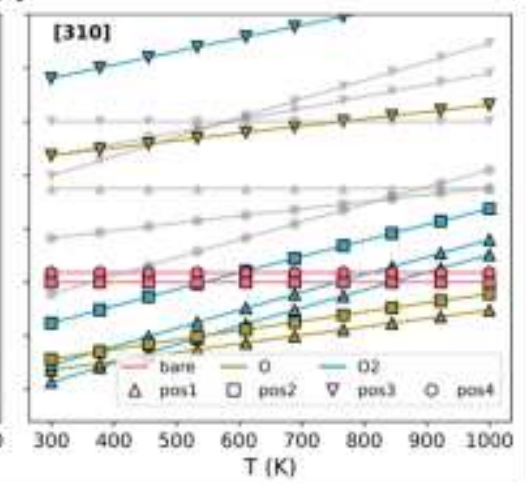


Figure 3. Atomistic models for the (a) [100], (b) [100] ${ }^{\mathrm{Mg}-\mathrm{vac}}$, and (c) [310] $\mathrm{MgO}$ facets considered in this work. Color scheme: $\mathrm{Mg}$ (green), O (red), Pt (gray). The corresponding temperaturedependent phase diagrams are summarized in (d), (e), and (f). Notation scheme for [100] and [100] ${ }^{\mathrm{Mg}-\mathrm{vac}} \mathrm{MgO}$ terraces: top surface (sub0), first (sub1), second (sub2), and third (sub3) subsurface layers. For the [310] facet, we considered various step site positions including leading step edge (pos1), lower step edge (pos2), underneath step edge (pos3), and behind step edge (pos4). The phase diagrams were calculated relative to the most-stable "bare" Pt-configuration (i.e., without adsorbates). All DFT-optimized structures are available as an Atomic Simulation Environment (ASE) database file ${ }^{25}$ in the group repository on GitHub.

The phase diagrams in Figure 3d-f show the relative stabilities of various Pt sites (i.e., locations and adsorbate coverages) for the [100], [100] ${ }^{\mathrm{Mg}-\mathrm{vac}}$, and [310] facets. Our calculations for the stoichiometric [100] and [310] surfaces show that surface Pt ([100]/sub0/* $\left.\mathrm{O}_{2}\right)$ and leading step edge $\left([310] / \operatorname{pos} 1 / * \mathrm{O}_{2}\right)$ sites are favored under our experimental conditions $\left(T=300 \mathrm{~K}, P_{O 2}=1\right.$ bar), respectively. Although the results demonstrate weaker $\mathrm{O}_{2}$ binding for $[100] / \mathrm{sub} 1\left(\mathrm{O}_{2}\right.$ desorption is predicted at $T>600 \mathrm{~K}$ ), the unsaturated [310]/pos1 (gold triangles) and [310]/pos2 (gold squares, $0.1 \mathrm{eV}$ less stable) sites are characterized by strong *O adsorption associated with the undercoordinated Pt atom. In contrast, no oxygen adsorption is predicted on the most stable $[100]^{\mathrm{Mg}-\mathrm{vac} / \mathrm{sub} 1}$ structure at room temperature.

Next, we compared the relative stabilities of Pt atoms at the surface and in sub-surface layers for the various $\mathrm{MgO}$ facets. Significantly, for the stoichiometric [100] facet, Pt sites in the first (second) sub-surface layer are $1.3(2.1) \mathrm{eV}$ less favorable energetically than those at the $\mathrm{MgO}$ surface (Figure 3d-f). Although surface Pt sites are preferred for the stoichiometric [100] surface (i.e., [100]/sub0, circles in Figure 3d), the calculations show that creation of a Mg-vacancy 
(denoted as $[100]^{\mathrm{Mg}-\mathrm{vac}}$ ) changes the relative stabilities. Specifically, in contrast to that in the [100] facet, Pt substitution in the first sub-surface layer $\left([100]^{\mathrm{Mg}-\mathrm{vac}} / \mathrm{sub} 1\right)$ is energetically more favorable than that in the surface layer $\left(0.8 \mathrm{eV}\right.$ less stable, $\left.[100]^{\mathrm{Mg}-\mathrm{vac}} / \mathrm{sub} 0\right)$ or the second sub-surface layer (0.3 eV less stable, $[100]^{\mathrm{Mg}-\mathrm{vac} / \mathrm{sub} 2)}$ (Figure 3d-f). These computational results are based on the PBESol functional; similar calculations with other functionals (RPBE/D3(BJ) and PBE/D3(BJ)) did not qualitatively change the trends (Figure S8).

\subsection{QuantEXAFS: Automated DFT-based Workflows for Fitting EXAFS Data with Improved Thoroughness and Objectivity.}

Although conventional EXAFS modeling indicates high $\mathrm{Pt}-\mathrm{O}$ and $\mathrm{Pt}-\mathrm{Mg} \mathrm{CNs}$ and the absence of a Pt-Pt contribution, we emphasize that this "hands-on" approach is limited to analyzing a few plausible, average scattering paths and does not identify a specific bonding configuration. This approach is therefore not sufficient to characterize the possible anisotropy of the individual $\mathrm{Pt}-\mathrm{O}$ and $\mathrm{Pt}-\mathrm{Mg}$ contributions. To overcome this limitation, we used the opensource X-ray analysis package Larch to perform EXAFS analysis of all 47 DFT-optimized structures mentioned above. ${ }^{26}$

The DFT-optimized structures were used "as-is" in the fitting, with fixed CNs and spatial orientations of $\mathrm{Mg}$ and $\mathrm{O}$ atoms in the fitting. In contrast to the typical approach whereby the average nearest-neighbors fits (described by the average bond lengths and CNs) are used to create structural models, we considered all relevant scattering paths (> 250 in some cases) for each DFToptimized structure. Notwithstanding the increased sophistication of the QuantEXAFS approach, it provides a typical EXAFS fit for each candidate structure in a few minutes, without user intervention. 


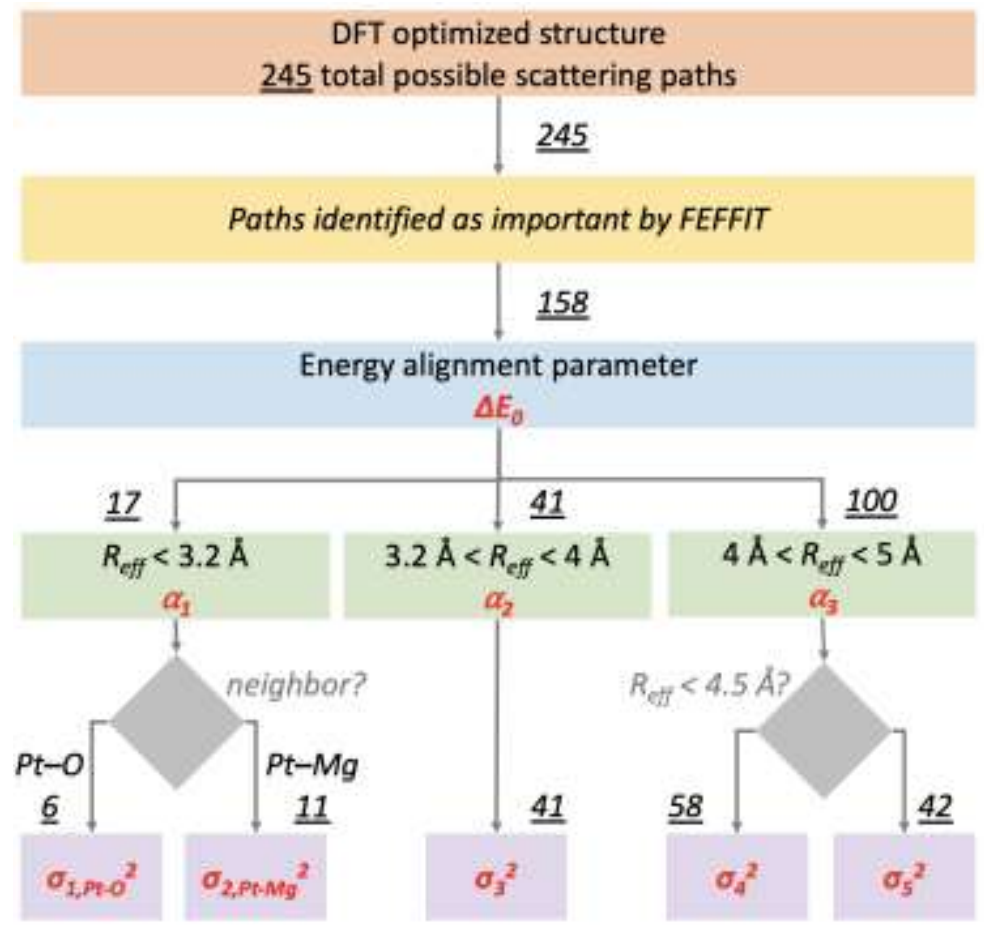

Figure 4. Flow-chart for hierarchically classifying all the possible paths into categories. The underlined numbers show the number of paths in the hierarchical classification approach, and the text in red identifies fitted parameters.

As the contribution of each scattering path to the total EXAFS depends on various parameters, it is important to limit the total number of variables in the fitting. This efficiency is achieved by (1) hierarchically classifying the individual paths into distinct categories based on their effective scattering distances $\left(R_{e f f}\right)$ and the identity of the scattering atom (if $R_{e f f}<3.2 \AA$ ) and (2) limiting the number of EXAFS variables optimized across each category. Specifically, for each DFT-optimized structure, our algorithm uses the following EXAFS fitting parameters:

(a) one energy alignment parameter (denoted as $\Delta E_{0}$ ); a single value is used for all paths

(b) three distance scaling parameters, $\alpha_{1}, \alpha_{2}$, and $\alpha_{3}$, one each for three different $R_{\text {eff }}$ ranges, where $R_{\text {eff,optimized }}=\alpha_{i} \times R_{e f f, D F T}$ and 
(c) five mean square variations in path lengths that depend on the scatterer identity (e.g., $\sigma_{1, P t-O}^{2}$ and $\sigma_{1, P t-M g}^{2}$ if $R_{e f f}<3.2 \AA$ ) and the effective scattering distance (e.g., $\sigma_{3}^{2}$, $\left.\sigma_{4}^{2}, \sigma_{5}^{2}\right)$

The hierarchical classification is summarized in Figure 4 and Table 1. The details of this approach and strategy of this categorization are discussed in the Supporting Information.

Table 1. Summary of hierarchical approach used to classify all possible scattering paths into five different categories. Only 9 parameters (shown in red) are optimized in the automated EXAFS analyses.

\begin{tabular}{|c|l|c|c|c|c|}
\hline \multirow{2}{*}{ Category } & Classification criterion & $\begin{array}{l}\text { Number } \\
\text { of paths }\end{array}$ & \multicolumn{3}{|c|}{ Variables } \\
\hline 1 & $R_{e f f}<3.2 \AA$ and Pt-O scatter & 6 & \multirow{2}{*}{$\alpha_{1}$} & $\sigma_{1, P t-O}^{2}$ \\
\hline 2 & $R_{e f f}<3.2 \AA$ and Pt-Mg scatter & 11 & & $\sigma_{1, P t-M g}^{2}$ \\
\hline 3 & $3.2 \AA<R_{e f f}<4 \AA$ & 41 & $\alpha_{2}$ & $\sigma_{3}^{2}$ \\
\hline 4 & $4 \AA<R_{e f f}<4.5 \AA$ & 58 & & $\alpha_{3}$ & $\sigma_{4}^{2}$ \\
\hline 5 & $4.5 \AA<R_{e f l}<5 \AA$ & 42 & & & $\sigma_{5}^{2}$ \\
\hline
\end{tabular}

The scattering path classification scheme described above ensures that (1) the constraints on the number of fitting parameters (calculated on the basis of the Nyquist criterion) are not violated and (2) physically relevant characteristics of scattering paths (e.g., different mean square variations in path length, that is, $\sigma_{1, P t-O}^{2}$ and $\sigma_{1, P t-M g}^{2}$ for $\mathrm{Pt}-\mathrm{O}$ and $\mathrm{Pt}-\mathrm{Mg}$ scatterers) are faithfully captured. The DFT structures serve as constraints making the EXAFS fitting more physically reasonable: They reduce the number of overall parameters that is needed for large number of paths that enables the modeling of the longer effective scattering distances $(\sim 5 \AA)$, including contributions from multiple scattering paths using distance-dependent fitting parameters (e.g., $\sigma_{i}^{2}$, and $\alpha_{i}$ ) without overfitting the data. These points are well-illustrated by our analyses for the 
$[100]^{\mathrm{Mg}-\mathrm{vac} / \mathrm{sub} 1}$ site (Figure 4), whereby only 9 parameters (Table 1 and 2) are used to model all 158 unique scattering paths.

More broadly, the classification scheme in Figure 4 creates a one-to-one mapping between a DFT-optimized structure and the resultant EXAFS fit. Specifically, we focus on the physical structure of the Pt site and not on the optimization of the averaged scattering paths. This is the central difference between the QuantEXAFS methodology and conventional EXAFS fitting.

Table 2. Optimized values of the 9 fitting parameters for the $[100]^{\mathrm{Mg}-\mathrm{vac}} / \mathrm{sub} 1$ site. Details of each individual scattering path are presented as a separate file in the SI.

\begin{tabular}{|c|c|c|c|c|}
\hline Category & $\Delta E_{0}(\mathrm{eV})$ & $\alpha_{i}$ & $10^{3} \times \sigma_{i}^{2}\left(\AA^{2}\right)$ & $\boldsymbol{R}_{\text {eff }, \text { optimized }}\left(\boldsymbol{A}^{2}\right)$ \\
\hline 1 & \multirow{5}{*}{$4.8 \pm 1.9$} & \multirow[b]{2}{*}{$0.994 \pm 0.005$} & $3.2 \pm 1.0$ & $2.01,2.01,2.02,2.03,2.04,2.05$ \\
\hline 2 & & & $6.7 \pm 1.6$ & $\begin{array}{c}2.96,2.97,2.98,2.98,2.99,2.99, \\
\quad 3.00,3.00,3.01,3.01,3.02\end{array}$ \\
\hline 3 & & $0.987 \pm 0.011$ & $6.3 \pm 7.0^{\alpha}$ & $3.40-3.79^{b}$ \\
\hline 4 & & \multirow{2}{*}{$0.996 \pm 0.001$} & $6.2 \pm 5.4^{a}$ & $4.02-4.40^{c}$ \\
\hline 5 & & & $5.3 \pm 4.3^{a}$ & $4.59-4.98^{d}$ \\
\hline
\end{tabular}

${ }^{a}$ Wide spans of $\sigma_{3}^{2}, \sigma_{4}^{2}, \sigma_{5}^{2}$ values arise as single and multiple scattering are considered together; ${ }^{b} 41$ Category 3 scattering paths; ${ }^{c} 58$ Category 4 scattering paths; ${ }^{d} 42$ Category 5 scattering paths.

The above fitting workflow was repeated for each unique DFT structure. In addition to the reduced $-\chi^{2}$ metric (commonly used by the XAS community), we quantified the agreement between the experimental and simulated EXAFS spectra for each DFT structure by using the Fréchet distance in $R$-space for the magnitude portions of the data and the model (denoted as $\delta_{\mathrm{F}}$, which measures the similarity between two curves). The histogram in Figure 5a shows the reduced- $\chi^{2}$ of the EXAFS fit corresponding to each DFT structure, along with the Fréchet distance (a lower value is better), corresponding to each structure (Table S6). In general, we observed that the $[100]^{\mathrm{Mg} \text {-vac }}$ structures (blue bars in Figure 5a) are in better agreement with experiment (reduced- $\chi^{2}<20, \delta_{\mathrm{F}}<$ 2). Although some of the [100] and [310] Pt configurations (e.g., [100]/sub1 and [310]/pos3/*O ${ }_{2}$ ) 
show satisfactory agreement with experimental EXAFS (reduced- $\chi^{2}<35, \delta_{\mathrm{F}}<4.5$ ), our DFT calculations show these configurations to be highly unstable $(1.5 \mathrm{eV}$ and $0.99 \mathrm{eV}$, respectively).
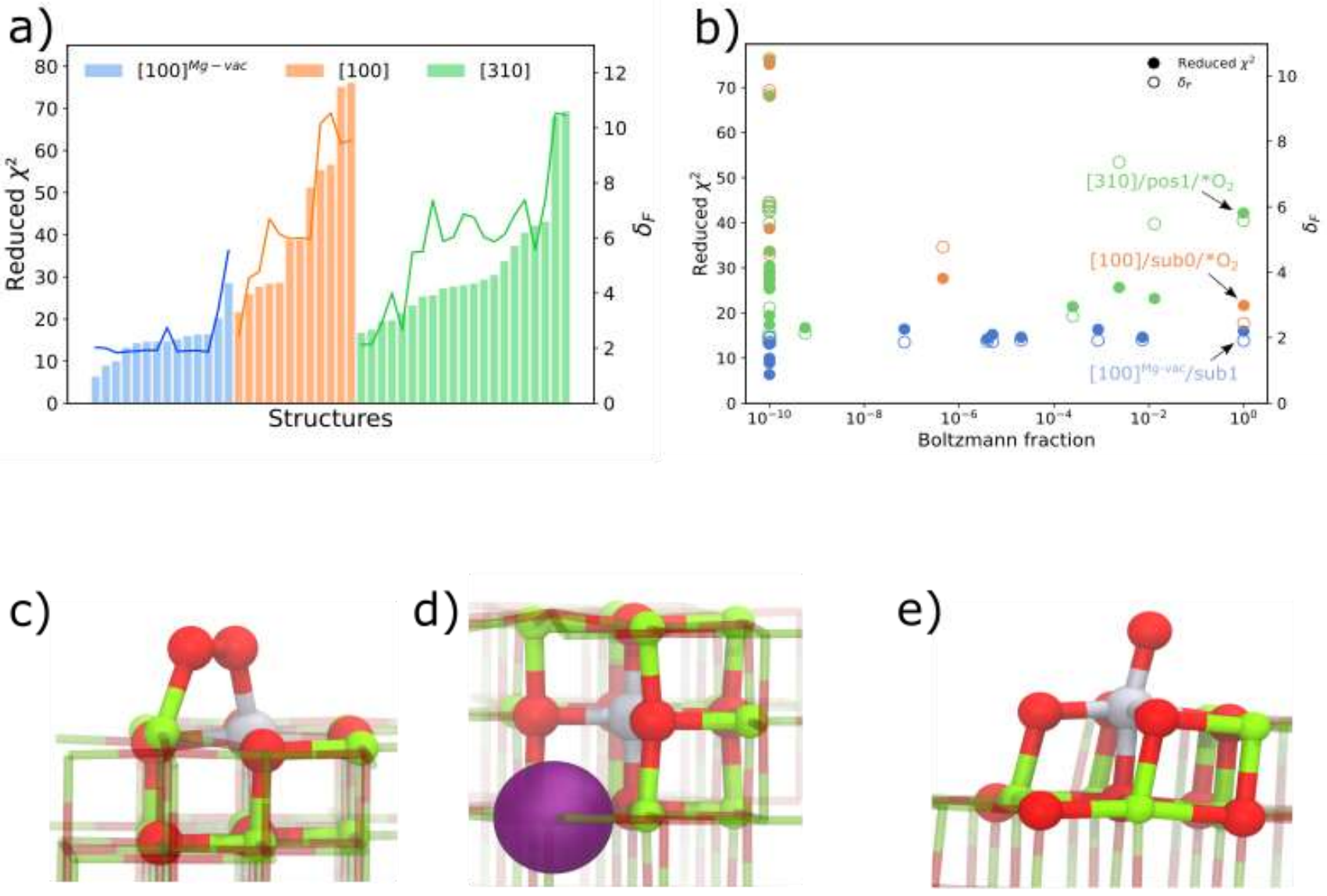

f) $[100] / \mathrm{sub}_{0} /{ }^{*} \mathrm{O}$,

g) $[100]^{\text {Mg_vac/sub1 }}$

h) $[310] / \operatorname{pos} 1 /{ }^{*} \mathrm{O}_{2}$
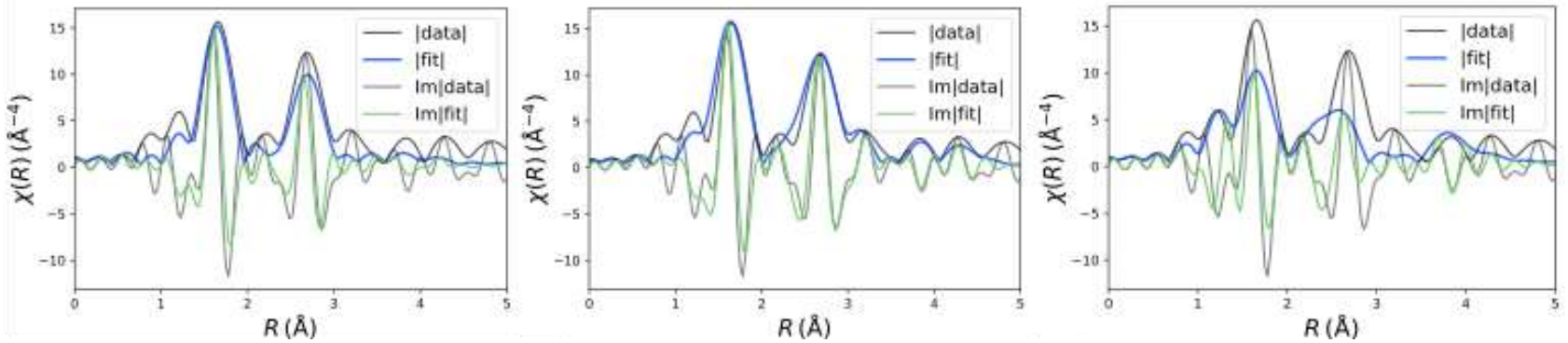

Figure 5. (a) Reduced- $\chi^{2}$ and Fréchet distance $\left(\delta_{F}\right)$ of EXAFS analysis for all the DFT-optimized structures, (b) Boltzmann fraction calculations for all facets. The DFT optimized geometries of the

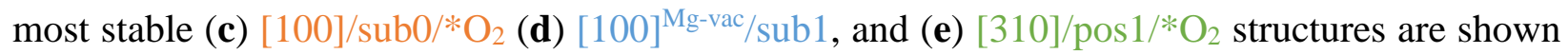


with the corresponding EXAFS fits in (f), (g), and (h) showing the magnitude (fit: blue, experiment: black) and imaginary portions (fit: green, experiment: black) of Fourier transforms. The $k$-range of $2.2-12.5 \AA^{-1}$ and the $R$-range of $1.0-5.0 \AA$ were used for the fits. Colors: $\mathrm{Mg}$ (green), O (red), Pt (grey). The purple sphere in (d) represents the sub-surface Mg-vacancy.

This interplay between thermodynamic stability of a structure and its consistency with the EXAFS data is quantitatively illustrated by comparing the quality of the EXAFS fits with the DFTcalculated Boltzmann fractions (at $300 \mathrm{~K}$ ) (Figure 5b). Unsurprisingly, only the most stable structures $\left([100] / \mathrm{sub0} / * \mathrm{O}_{2}\right.$ (orange circle), $[100]^{\mathrm{Mg}-\mathrm{vac}} / \mathrm{sub} 1$ (blue triangle), and $[310] / \mathrm{pos} / *^{*} \mathrm{O}_{2}$ (green triangle)) were observed in any significant number (based on Boltzmann distributions). The EXAFS fits corresponding to the three stable Pt configurations (Figure 5c-e) are presented in Figure 5f-h, showing that the $[100]^{\mathrm{Mg}-\mathrm{vac}} / \mathrm{sub} 1\left(\mathrm{CN}_{\mathrm{Pt}-\mathrm{O}}=6, \mathrm{CN}_{\mathrm{Pt}-\mathrm{Mg}}=11\right)$ model agrees most closely with experiment. Specifically, although the $[100] / \mathrm{sub} / * \mathrm{O}_{2}$ structure agrees satisfactorily with the $\mathrm{Pt}-\mathrm{O}$ scattering path $\left(\mathrm{CN}_{\mathrm{Pt}-\mathrm{O}}=7\right)$, the $\mathrm{Pt}-\mathrm{Mg}$ path is underestimated, corresponding to the much lower Pt-Mg contribution $\left(\mathrm{CN}_{\mathrm{Pt}-\mathrm{Mg}}=8\right)$. We emphasize that the [310]/pos3 site (as suggested by

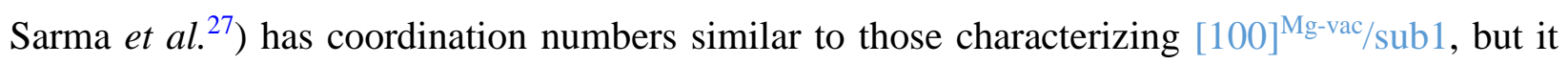
does not agree well with the EXAFS data (Figure S10). These results demonstrate that both the effects of coordination number (i.e., $[100] / \mathrm{subO}^{*} \mathrm{O}_{2}$ vs. $[100]^{\mathrm{Mg}-\mathrm{vac} / \mathrm{sub} 1)}$ ) and the local spatial

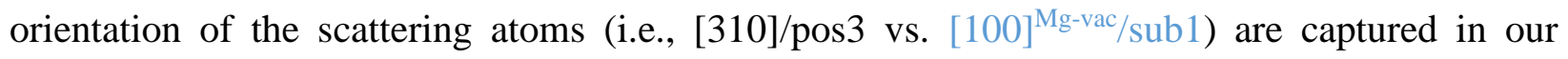
EXAFS fitting approach.

At this stage, it is useful to highlight an additional advantage of our EXAFS fitting procedure, with $[100]^{\mathrm{Mg}-\mathrm{vac} / \mathrm{sub} 1}$ used as an illustrative example. Instead of using the average Pt$\mathrm{O}$ (and/or $\mathrm{Pt}-\mathrm{Mg}$ ) distances and coordination numbers as the fitting parameters (as done 
conventionally), in our approach each individual Pt-O (total 6 for $[100]^{\mathrm{Mg}-v a c} / \mathrm{sub} 1$ as an example) and $\mathrm{Pt}-\mathrm{Mg}$ (total 11) scattering path is considered separately. This procedure allows us to capture the anisotropies of the local bonding environment, as indicated by the DFT-calculated bond lengths. Overall, we considered 158 total paths, which included single, double, and multiple scattering paths (involving up to 5 scattering atoms), using only use 9 fitting variables (Tables 1 and 2) to achieve unprecedented agreement with the experimental EXAFS across the entire $R$ range (Figure 5g).

As the entire workflow relies on physically motivated models, it is possible to bolster the selfconsistency between DFT structures and EXAFS fits. Beyond providing good models for the EXAFS fits, the DFT calculations also provide force constants that can be used to generate abinitio vibrational mean-square relative displacements for the EXAFS (i.e., $\sigma^{2}$ ). ${ }^{28-30}$ Specifically, the values for $\sigma^{2}$ for Pt-Mg $\left(0.005 \AA^{2}\right)$ and $\mathrm{Pt}-\mathrm{O}\left(0.003 \AA^{2}\right)$ obtained from DFT-calculated harmonic frequencies are consistent with the results of the EXAFS fitting (Pt-Mg: $0.0067 \pm 0.001$ $\AA^{2}$, Pt-O: $0.0032 \pm 0.001 \AA^{2}$ ) (Table S7). This point highlights the uniqueness of our approach: (1) the DFT-optimized structures are used to perform an objective, thorough EXAFS fitting, and (2) the EXAFS fitting parameters (themselves determined using the automated Python workflows) are consistent with independently calculated thermal disorders (from DFT vibrations). This level of detail has not been reported for any atomically dispersed catalysts, and we posit that it represents a significant step forward in automated analysis, self-consistent interpretation of EXAFS data.

\subsection{XANES Evidence of Structure.}

In addition to the EXAFS, we also consider the XANES data (Figure S11; data characterizing reference compounds are shown for comparison). XANES spectra provide evidence 
of the Pt electronic structure, as the intensity of the white line at the $\mathrm{Pt} \mathrm{L}_{3}$ edge is a measure of the unoccupied d-states and occupied p-states $\left(2 p_{3 / 2} \rightarrow 5 d_{3 / 2}\right.$ or $\left.5 d_{5 / 2}\right) .{ }^{31}$ The oxidation state of the supported $\mathrm{Pt}$ is close to that of $\mathrm{Pt}^{4+}$ in $\mathrm{H}_{2} \mathrm{Pt}(\mathrm{OH})_{6}$ and markedly different from that of the $\mathrm{Pt}^{2+}$ in $\left(\mathrm{Pt}(\mathrm{acac})_{2}\right)\left(\mathrm{acac}=\right.$ acetylacetonato, $\left.\left[\mathrm{C}_{5} \mathrm{H}_{7} \mathrm{O}_{2}\right]^{-}\right)$and $\mathrm{Pt}^{0}$ in $\mathrm{Pt}$ foil (Figure S11). These data imply that isolated $\mathrm{Pt}^{4+}$ was present in six-coordinate octahedral geometry in cation vacancy sites, consistent with the STEM and EXAFS data. To account for charge balance, one $\mathrm{Pt}^{4+}$ in an $\mathrm{MgO}$ sub-surface site requires one $\mathrm{Mg}^{2+}$ vacancy, which implies a Pt-Mg coordination number of 11 and not 12, consistent with the EXAFS analyses. These results further agree with DFT-calculated Bader charges that show consistent oxidation states for the sub-surface $\mathrm{Pt}^{4+}$ with one $\mathrm{Mg}$ vacancy and the $\mathrm{Pt}^{4+}$ in $\mathrm{H}_{2} \mathrm{Pt}(\mathrm{OH})_{6}\left(q_{\mathrm{Bader}}=+1.35 \mathrm{e}\right.$ and $+1.41 \mathrm{e}$, respectively) (Table S8). These values are much higher than $q_{\text {Bader }}=+0.86 \mathrm{e}$ for the $\mathrm{Pt}^{2+}$ in $\mathrm{Pt}(\mathrm{acac})_{2}\left(\right.$ Table S8). ${ }^{32}$
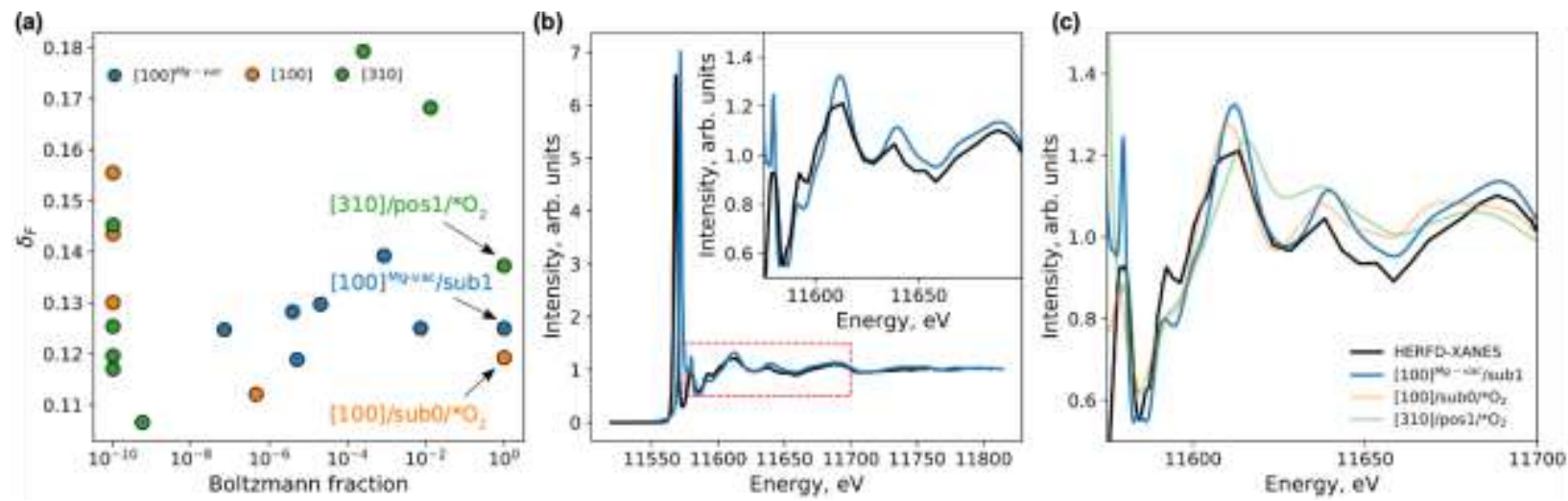

Figure 6. (a) Fréchet distances from comparison of HERFD-XANES data with FEFF-simulated XANES and DFT-calculated Boltzmann fractions. (b) Simulated and experimental XANES

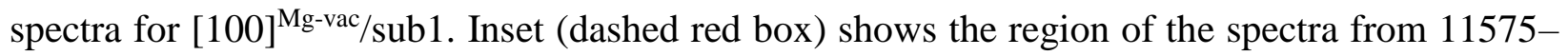
$11700 \mathrm{eV}$. (c) Comparison of FEFF-simulated XANES spectra of the most stable structures from each facet $\left([100] \mathrm{Mg}\right.$-vac/sub1, $[100] / \mathrm{sub} / * \mathrm{O}_{2}$, and $\left.[310] / \mathrm{pos} 1 / * \mathrm{O}_{2}\right)$ with HERFD-XANES data from $11575-11700 \mathrm{eV}$. 
We also collected HERFD-XANES spectra; HERFD-XANES minimizes the limitations of energy resolution affected by core-hole lifetime broadening and beamline optics in conventional XANES. ${ }^{33}$ A comparison of the HERFD and conventional XANES data (Figure S11) shows the advantages of HERFD in resolving near-edge features including (a) the stable near-zero signal in the pre-edge; (b) strong intensities and sharpness without a long tail characterizing the white line; and (c) the clear resonance feature with little broadening beyond the white line.

The HERFD-XANES data provide insight into the catalyst structure beyond what is determined by the EXAFS data and DFT-guided analysis. ${ }^{16}$ The HERFD-XANES data were compared with FEFF-simulated XANES spectra based on the aforementioned DFT-determined structures (Figure 6). ${ }^{34,35}$ The agreement between experimental and simulated XANES spectra (quantified using the Fréchet distance $\left(\delta_{\mathrm{F}}\right)$ ) is compared with the thermodynamic stability (Figure 6a) of various Pt sites. Figure 6b, c show a comparison of the experimental HERFD and the FEFF simulations for each of the most stable structures predicted from DFT-calculated Boltzmann fractions: $[100] / \mathrm{sub} / * \mathrm{O}_{2}(99.2 \%),[100]^{\mathrm{Mg}-\mathrm{vac}} / \mathrm{sub} 1(99.9 \%)$, and $[310] / \mathrm{pos} 1 / * \mathrm{O}_{2}(98.4 \%)$. The FEFF results were shifted $4.5 \mathrm{eV}$ and scaled to match the normalized experimental results for values $>11650 \mathrm{eV}$. Of all the stable structures, both $[100] / \mathrm{sub} 0 / * \mathrm{O}_{2}$ and $[100]^{\mathrm{Mg}-v a c} / \mathrm{sub} 1$ show good agreement with the experimental XANES; however, by comparing the corresponding EXAFS spectra in Figure $5 \mathrm{f}$ and $\mathrm{g}$, we see that the Mg-vacancy provides a notable improvement over $[100] / \mathrm{sub} 0 / * \mathrm{O}_{2}$. Although some [310] structures are more consistent with experiment than these $\left(\delta_{\mathrm{F}}<0.125\right)$, they are significantly less stable (Boltzmann fraction $\left.<10^{-9}\right)$, and, we infer, unlikely to exist. The most stable [310] structures show poor agreement with experiment (Figure 6c (green)). 
Taking together the unique combination of EXAFS and HERFD-XANES fits of $[100]^{\mathrm{Mg}-}$ vac/sub1 and the DFT-predicted stability, our results show that the Pt/MgO catalyst is wellcharacterized as an atomically dispersed $\mathrm{Pt}^{+4}$ ion embedded in the first subsurface layer (sub1) located adjacent to a Mg-vacancy site. As shown in Figure S12, our HERFD-XANES analyses and DFT calculations do not identify the exact location of the Mg-vacancy site; we used the most stable structure, Mg-vacancy in the surface layer (sub0) for further analysis.

To analyze the origin of the various features in the HERFD, Figure S13 shows the locally projected, $l$-dependent density of states $\left(l\right.$-DOS) of the $[100]^{\mathrm{Mg}-\mathrm{vac}} / \mathrm{sub} 1$ site. Overall, the Pt $\mathrm{L}_{3}$ HERFD spectra resemble the Pt $d$-DOS, as indicated by the vertical dashed lines. The white line is composed largely of $\mathrm{Pt} d$-DOS with a small contribution from the $\mathrm{O} p$-DOS, with its position being largely insensitive to the local disorder. The second peak ( $\sim 9 \mathrm{eV}$ above the Fermi level) shows contributions from both the $\mathrm{O}$ and $\mathrm{Mg}$ atoms as well as the local $\mathrm{Pt} s$-DOS, indicating that this feature corresponds to non-local states and arises at least in part from bonding of Pt to the near-neighboring atoms (Figure S13).

\subsection{CO Oxidation Catalyzed by Pt/MgO.}

We characterized the $\mathrm{Pt} / \mathrm{MgO}$ as a catalyst for $\mathrm{CO}$ oxidation as a probe reaction. The lightoff curves (Figure 7a) show that the reaction became readily measurable at approximately $180{ }^{\circ} \mathrm{C}$, with the $\mathrm{CO}$ conversion reaching nearly $100 \%$ at $280{ }^{\circ} \mathrm{C}$ under our conditions. Separate experiments were carried out to determine reaction rates (turnover frequencies, TOF, rates per Pt atom) from data obtained at low conversions $(<5 \%)$, which were shown to be differential by the linear dependence of conversion on inverse space velocity at temperatures of $180-200{ }^{\circ} \mathrm{C}$ (Figure 7b,c). An Arrhenius plot (Figure 7d) indicates an apparent activation energy of $79 \pm 2 \mathrm{~kJ} \mathrm{~mol}^{-1}$. Data showing conversion as a function of time onstream in the flow reactor under steady-state 
conditions (Figure S14) indicate that the catalyst was stable, retaining its activity for as long as it was onstream (48 h) at $210^{\circ} \mathrm{C}$. The catalyst remained white in color after use. The HAADF-STEM images of used $\mathrm{Pt} / \mathrm{MgO}$ samples (after three light-off experiments) show that $\mathrm{Pt}$ was still atomically dispersed (Figure S15), even at a high temperature $\left(300{ }^{\circ} \mathrm{C}\right)$. As further checks of the catalyst stability, we recorded in-operando HERFD-XANES data during CO oxidation at $210{ }^{\circ} \mathrm{C}$ (Figure S16), demonstrating unchanged spectra.
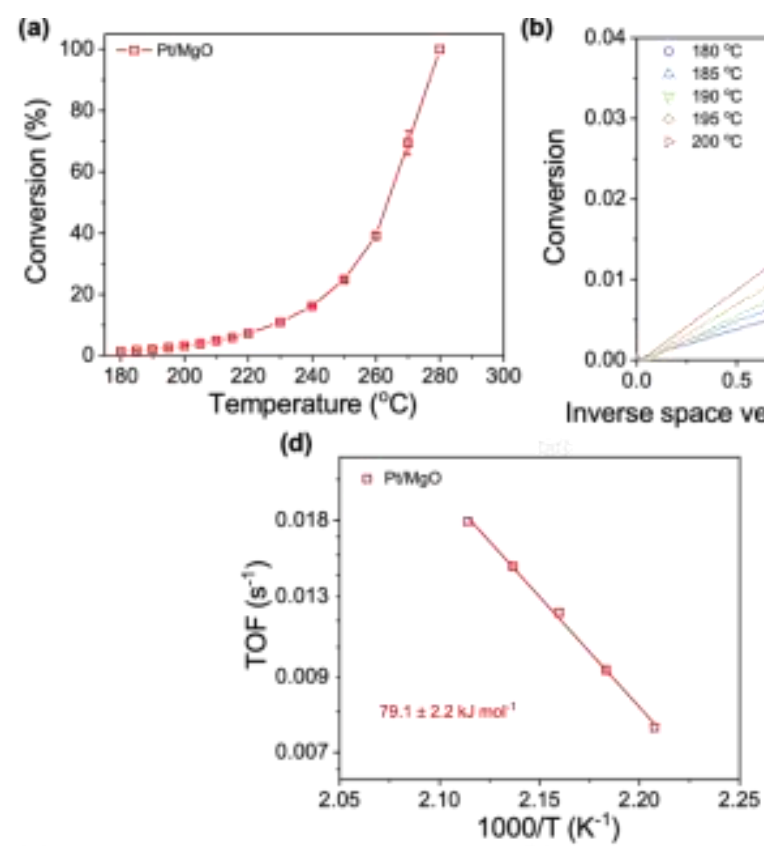

(b)

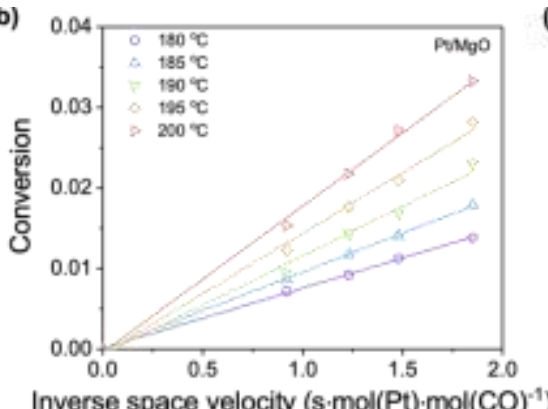

(e)

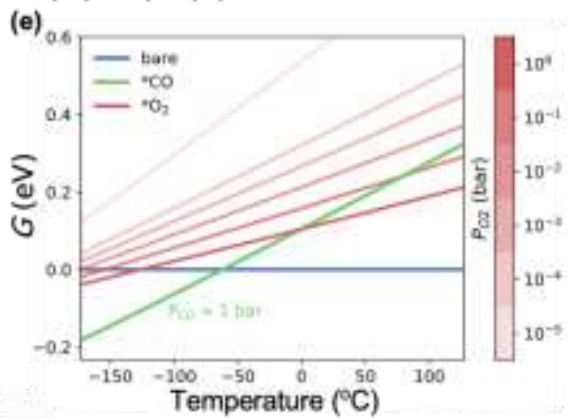

Figure 7. (a) Light-off curve characterizing $\mathrm{CO}$ oxidation catalyzed by $\mathrm{Pt} / \mathrm{MgO}$. Error bars represent standard deviation determined from three independent measurements. The once-through plug-flow reactor was heated from room temperature at a rate of $2{ }^{\circ} \mathrm{C} / \mathrm{min}$ to $300{ }^{\circ} \mathrm{C}$. The feed was $5.0 \% \mathrm{CO}$ in helium flowing at $4.0 \mathrm{~mL}(\mathrm{NTP}) / \mathrm{min}+5.0 \% \mathrm{O}_{2}$ in helium flowing at $16.0 \mathrm{~mL}$ (NTP)/min; the catalyst mass was $100 \mathrm{mg}$. (b) Demonstration of differential conversion at various temperatures. Low-conversion $(<5 \%)$ data were obtained under the conditions stated in a, except that the feed gas was $5.0 \% \mathrm{CO}$ in helium flowing at 5.0, 6.0, or $8.0 \mathrm{~mL}(\mathrm{NTP}) / \mathrm{min}+5.0 \% \mathrm{O}_{2}$ in helium flowing at 20.0, 24.0, or $32.0 \mathrm{~mL}$ (NTP)/min; the catalyst mass was $100 \mathrm{mg}$. (c) TOF values 
determined from slopes of lines in b, with errors. (d) Arrhenius plot. (e) DFT-calculated CO and $\mathrm{O}_{2}$ adsorption at various temperatures and $\mathrm{O}_{2}$ partial pressures.

This catalytic probe reaction provided yet another opportunity to verify the identity of the support sites for Pt. Figure 7e shows trends in CO adsorption for the stable $[100]^{\mathrm{Mg} \text {-vac/sub1 Pt }}$ terrace site at various temperatures. Unsurprisingly, stronger $\mathrm{CO}$ adsorption was found for the undercoordinated Pt site (i.e., [100]/sub0); we predicted CO adsorption at temperatures as high as about $83{ }^{\circ} \mathrm{C}$ (Figure S17). In contrast, the sub-surface Pt site ([100 $]^{\mathrm{Mg}-\mathrm{vac} / \mathrm{sub} 1)}$ is characterized by weaker $\mathrm{CO}$ binding $\left(0.25 \mathrm{eV}\right.$ at $\left.83^{\circ} \mathrm{C}\right)$; no $\mathrm{CO}$ adsorption was predicted at room temperature. The trends for the $[100]^{\mathrm{Mg}-\mathrm{vac}} / \mathrm{sub} 1$ site are consistent with (1) our IR experiments that do not show significant CO uptakes at room temperature (Figure S18) and (2) Sarma's report ${ }^{27}$ of lowtemperature $\left(-163{ }^{\circ} \mathrm{C}\right)$ IR spectra of $\mathrm{CO}$ on a sample similar to ours; a $2166 \mathrm{~cm}^{-1} v_{\mathrm{CO}}$ band was observed, agreeing within error with our calculated frequency $\left(2141 \mathrm{~cm}^{-1}\right)$.

It is significant, and a challenge to our interpretation, that our $\mathrm{Pt} / \mathrm{MgO}$ catalyst, which consists of sub-surface, highly coordinated Pt sites, does not bind $\mathrm{CO}$ at room temperature but nonetheless is catalytically active for $\mathrm{CO}$ oxidation. Thus, we turned to DFT calculations (RPBE/D3-BJ functional, nudged elastic band and dimer method for barriers) to investigate various possible mechanisms of $\mathrm{CO}$ oxidation on the $[100]^{\mathrm{Mg}-\mathrm{vac}} / \mathrm{sub} 1$ site, and the two most favorable mechanisms are shown in Figure 8. Indeed, our calculations show that the insertion of $\mathrm{CO}$ into the surface-bound $\mathrm{O}_{2}$ is the rate limiting step - the calculated enthalpic barrier (107.4 $\mathrm{kJ} / \mathrm{mol}$ ) is in satisfactory agreement with experimentally determined apparent activation energies (Figure 8a); similar carbonate species were proposed by Sarma et al. ${ }^{27}$ for Pt at the step site on $\mathrm{MgO}$. The subsurface $\mathrm{Pt}$ and $\mathrm{Mg}$ vacancy activate the bound $\mathrm{O}_{2}$ and allow for $\mathrm{CO}$ insertion to form 
the intermediate carbonate. Charge density difference plots showing the change in the electronic structure of the $* \mathrm{O}_{2}$ compared with that on defect-free crystalline $\mathrm{MgO}$ are shown in Figure S19. Although the role of the Pt atom in this mechanism differs markedly from what has been reported by Sarma et al., ${ }^{27}$ the calculated free energy barrier at $300{ }^{\circ} \mathrm{C}$ in this work is slightly lower (169 $\mathrm{kJ} / \mathrm{mol}$ compared with $173 \mathrm{~kJ} / \mathrm{mol}^{27}$ ). Moreover, a similar Eley-Rideal mechanism has been inferred for an $\mathrm{Ag} / \mathrm{MgO}$ catalyst. ${ }^{36}$ A second mechanism (similar to that presented by Sarma et al.) consisting of the abstraction of a surface oxygen by $\mathrm{CO}$ to form an oxygen vacancy as the rate limiting step is shown in Figure 8b. The similarity of the free energy barriers of these two mechanisms (170.2 kJ/mol vs $168.8 \mathrm{~kJ} / \mathrm{mol})$ makes it challenging to discern which should be favored and both are plausible according to our calculations.
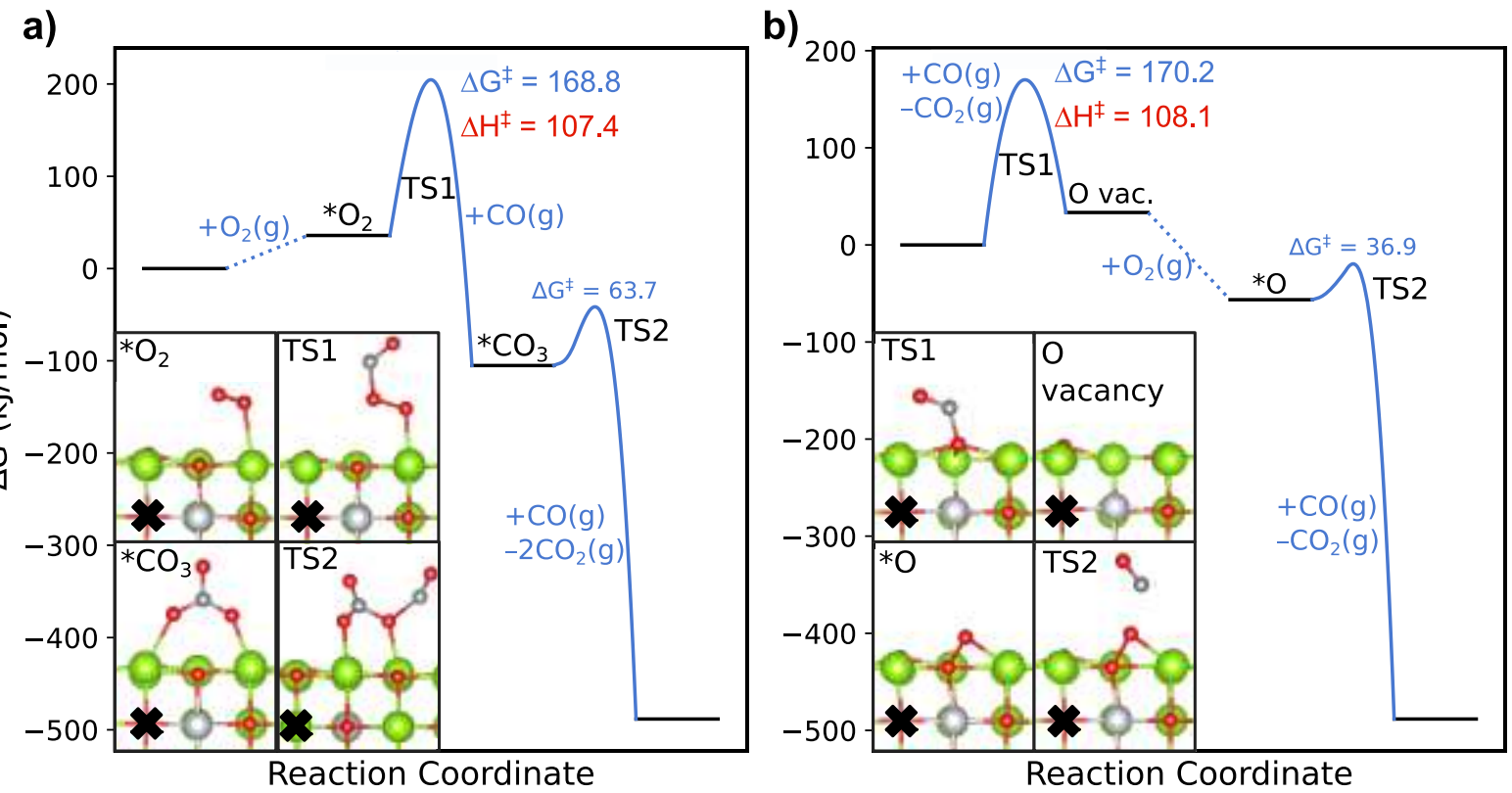

Figure 8. Reaction free energy diagrams for $\mathrm{CO}$ oxidation on $[100]^{\mathrm{Mg}-\mathrm{vac}} / \mathrm{sub} 1$ surface at $300{ }^{\circ} \mathrm{C}$. Mechanism a) is an Eley-Rideal mechanism with the formation of a carbonate intermediate, and mechanism b) proceeds through the formation of an oxygen vacancy above the subsurface Pt. Calculated free energy (blue) and enthalpic (red) barriers are presented for each transition state. 
Insets show the optimized structures of the key intermediate species with the location of the $\mathrm{Mg}$ vacancy marked with a black cross (colors: $\mathrm{Mg}$, green; $\mathrm{O}$, red; $\mathrm{Pt}$, light gray; $\mathrm{C}$, dark gray).

At this stage, it is useful to examine whether the $\mathrm{CO}$ oxidation reaction affects the $\mathrm{Pt}$ bonding sites. Specifically, as the EXAFS data represent an average of all the Pt environments, we posit that our EXAFS workflow is sufficiently versatile to provide quantitative characterization of the heterogeneity of the Pt sites (Figure S20). As a demonstration, we simultaneously fitted two Pt

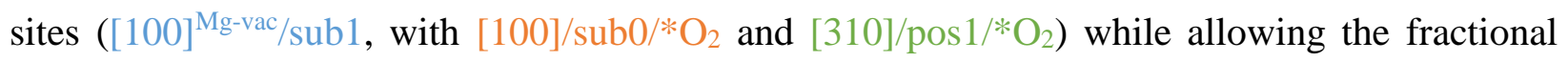
contribution of each site and the EXAFS fitting parameters to vary simultaneously. The results, summarized in Figure S21, show that $[100]^{\mathrm{Mg}-\mathrm{vac}} / \mathrm{sub1}$ is the dominant site for the as-synthesized ( $71.1 \%$ of total $\mathrm{Pt})$ and the used $(80.3 \%$ of total $\mathrm{Pt})$ catalysts. Indeed, we observed that although the fraction of the major $[100]^{\mathrm{Mg}-\mathrm{vac}} / \mathrm{sub} 1$ site did not change significantly during catalysis, the contributions of the minority species ([100]/sub0/* $\mathrm{O}_{2}: 28.9 \%$ (fresh), $19.7 \%$ (used) did change). The contributions of the $[310] / \operatorname{pos} 1 / * \mathrm{O}_{2}$ site were negligible in both cases. Detailed discussion of this quantitative EXAFS fitting is beyond the scope of this investigation.

\section{DISCUSSION}

The intense recent attention paid to atomically dispersed supported metal catalysts reflects the promise of catalysts with valuable new properties and the prospect that some will be so simple in structure as to propel significant advances in the understanding of surface catalysis broadly at the atomic level. ${ }^{9,37}$ Some literature reflects the notion that these catalysts are straightforward to understand because the metals are atomically dispersed ${ }^{37}$, but this view overlooks the intrinsic complexity of the supports and the need to incorporate the metal-support combinations in realistic 
models of the catalysts. Thus, there is a motivation to work with metal-support-site combinations that are nearly unique and can be understood in depth. Consequently, researchers have been motivated to use crystalline metal oxides as supports and to use low metal loadings to create catalysts that can be approximated as isolated metals in unique, stable surroundings.

The characterization data presented here validate that approach. They have a high degree of internal consistency and show that the structure of the catalyst consisting of Pt in a loading of only $0.05 \mathrm{wt} \%$ on crystalline $\mathrm{MgO}$ powder is well represented with a single, stably encapsulatedyet still catalytically active - structure that meets the criterion of near structural uniqueness. A full set of complementary experimental characterizations combined with theoretical verification was needed to test the hypothesis that such a catalyst could be characterized structurally with some confidence.

The DFT-guided EXAFS fitting approach described here represents, we posit, a substantial advance in the characterization of such catalysts. Although previous approaches represent attempts to develop DFT models that are motivated by results of conventional EXAFS analyses, we instead used a library of DFT-optimized Pt structures to identify the single best representation of the all the experimental results (e.g., EXAFS, HERFD-XANES, IR spectra of adsorbed CO, and catalytic kinetics) collected in this investigation.

Going forward, we anticipate that further development of this combination of characterization techniques will help guide the choice of metal-support combinations to advance the field of atomically dispersed supported metal catalysts; help advance the understanding of the broad class of supported catalysts; and help guide the development of new and improved methods for understanding of even more complex surface structures.

\section{CONCLUSIONS}


The methodology used in the fitting of EXAFS data (denoted as QuantEXAFS) is performed with a library of DFT structures that are grounded in the known structure of crystalline MgO. The physically motivated, DFT-optimized structures are a significant improvement over today's conventional EXAFS fitting procedures, which usually rely on bond distances for nominally comparable reference compounds for initial estimates of scattering distances. The conventional EXAFS analysis relies on the fitting of a few, average scattering paths, which may not be representative of physically motivated structures. A central point about the method reported here is the one-to-one mapping between a DFT-optimized structure and the resultant EXAFS fit. The focus of the method is on the physical structure and not the individual scattering paths. We emphasize that there is more to using DFT structures than just positions of atoms in space; we have placed the stability of the structure (i.e., the Boltzmann distribution) at the center of the analysis. Our automated analysis and the path classification scheme allow efficient implementation of hundreds of unique paths, implying perhaps an order of magnitude improvement in the accuracy and reliability of the analysis compared with conventional analyses. The workflow presented in this work removes potential user bias from the EXAFS modeling. We realize that the methods rest on the foundation of structural knowledge of the support-which is crystalline, with defects. A limitation of the method is that such a firm structural foundation is not available for many catalytic materials, such as amorphous supports, and the challenge of extending the methodology to these more complex materials — and accounting for their heterogeneity_is formidable. 


\section{EXPERIMENTAL AND COMPUTATIONAL METHODS}

Synthesis of Pt/MgO Samples. MgO powder (1000 mg, US Research Nanomaterials) with a manufacturer-specified specific surface area of $25 \mathrm{~m}^{2} / \mathrm{g}$ was dispersed in $250 \mathrm{~mL}$ of ethanol in a 500-mL beaker with magnetic stirring. Aqueous $\mathrm{K}_{2} \mathrm{PtCl}_{4}, 20 \mathrm{~mL}$ of $0.25 \mathrm{mM}$ solution, and $40 \mathrm{~mL}$ of ethanol were transferred into the stirred beaker through a syringe pump at a rate of $5 \mathrm{~mL} / \mathrm{h}$. The resultant solid was collected by centrifugation, washed with distilled water, and then dried overnight in air in an oven at $80{ }^{\circ} \mathrm{C}$. The resultant powder was calcined in flowing $\mathrm{O}_{2}(10$ $\mathrm{mL}(\mathrm{NTP}) / \mathrm{min})$ and $\mathrm{N}_{2}(40 \mathrm{~mL}(\mathrm{NTP}) / \mathrm{min})$ as the temperature was ramped from room temperature to $120^{\circ} \mathrm{C}$ and held for $2 \mathrm{~h}$ to remove any residual water and organics. The sample was then further calcined in a mixture of flowing $\mathrm{O}_{2}(10 \mathrm{~mL}(\mathrm{NTP}) / \mathrm{min})$ and $\mathrm{N}_{2}(40 \mathrm{~mL}(\mathrm{NTP}) / \mathrm{min})$ as the temperature was ramped at a rate of $5{ }^{\circ} \mathrm{C} / \mathrm{min}$ until a final temperature of $700{ }^{\circ} \mathrm{C}$ was reached, which was held for $4 \mathrm{~h}$.

X-ray Absorption Spectroscopy. XAS was carried out at beamlines 4-1 and 6-2 at the Stanford Synchrotron Radiation Lightsource (SSRL). At beamline 4-1, which is a side station on a 20-pole wiggler beamline, the ring SPEAR3 was operated in top-off mode with a storage ring energy of 3 $\mathrm{GeV}$ and $500 \mathrm{~mA}$ stored current. A Si double-crystal (220) monochromator was detuned by 20 $25 \%$ of maximum intensity to minimize harmonics at the $\mathrm{Pt}_{3}$ edge. XAS data were collected in fluorescence yield mode with a 30-element germanium solid-state detector array with samples pressed into a pellet at $25^{\circ} \mathrm{C}$. For energy calibration, a Pt foil reference was placed between the ion chambers upbeam and downbeam of the sample so that its spectrum was measured simultaneously with that of the sample.

At beamline 6-2, which is fed by a 56-pole, 0.9 Tesla wiggler, high-energy resolution fluorescence detection HERFD XANES experiments were conducted with a liquid-nitrogen- 
cooled double-crystal Si (311) monochromator to select the energy of the incident beam. A Rowland circle spectrometer (radius $1 \mathrm{~m}$ ) equipped with three spherically bent $\mathrm{Si}(800)$ analyzers and a silicon drift detector were used to select the Pt- $\mathrm{L}_{\alpha}$ emission line. A Pt foil was scanned in the transmission mode for initial energy calibration. For ex-situ HERFD-XANES experiments, the sample was pressed into a pellet at $25^{\circ} \mathrm{C}$. In each in-operando HERFD-XANES experiment, approximately $50 \mathrm{mg}$ of catalyst sample was loaded into a flow-through cell, a Kapton tube (i.d. = $2.8 \mathrm{~mm}$ ), connected to a treatment gas line. ${ }^{38}$ The compositions of effluent gases flowing from the cell were measured with an online mass spectrometer (Hiden HPR20). The Pt/MgO was first heated in helium flowing at $20 \mathrm{~mL}(\mathrm{NTP}) / \mathrm{min}$ as the temperature was ramped from room temperature to $210^{\circ} \mathrm{C}$ at a rate of $5^{\circ} \mathrm{C} / \mathrm{min}$. Then the feed gas was switched to a mixture of $\mathrm{CO}$ flowing at $0.2 \mathrm{~mL}(\mathrm{NTP}) / \mathrm{min}+\mathrm{O}_{2}$ flowing at $0.8 \mathrm{~mL}(\mathrm{NTP}) / \mathrm{min}+$ helium flowing at 19 $\mathrm{mL}(\mathrm{NTP}) / \mathrm{min}$ with the sample in the cell held at $210{ }^{\circ} \mathrm{C}$ for $1 \mathrm{~h}$ as $\mathrm{CO}$ oxidation catalysis took place. Thereafter, the reactor was cooled to room temperature and the gas feed switched to helium flowing at $20 \mathrm{~mL}(\mathrm{NTP}) / \mathrm{min}$. HERFD-XANES spectra were collected periodically during all these steps.

IR Spectroscopy. Transmission IR spectra of powder samples in the $v_{O-} \mathrm{H}_{\text {region were determined }}$ with a Bruker IFS 66v/S spectrometer with a resolution of $4 \mathrm{~cm}^{-1}$. Approximately $10 \mathrm{mg}$ of sample was loaded between two $\mathrm{KBr}$ windows, and spectra were recorded at room temperature with the sample under vacuum, with an average of 256 scans per spectrum. IR spectra of samples with adsorbed $\mathrm{CO}$ were determined with approximately $40 \mathrm{mg}$ of sample pressed into a wafer loaded into a cell (In-situ Research Instruments, South Bend, IN) that served as a flow reactor fed with various gases, and transmission spectra of catalysts in the presence of these gases were recorded with a liquid-nitrogen-cooled MCT detector. 
CO Oxidation Catalysis in a Conventional Laboratory Plug-flow Reactor. The catalyst samples were evaluated for $\mathrm{CO}$ oxidation in a once-through plug-flow reactor, with products analyzed with an online mass spectrometer (Hiden Analytical HPR20) equipped with a secondary electron multiplier detector used in multiple ion detection mode. Samples of catalyst powder (100 mg) that had passed through a 40 to 60 mesh sieve were loaded into a quartz tube reactor (i.d. $=4$ $\mathrm{mm}$ ), with the upstream and downstream sections packed with quartz wool. In experiments to determine light-off curves for $\mathrm{CO}$ oxidation, the feed was a mixture of 5.0\% $\mathrm{CO}$ in helium flowing at $4.0 \mathrm{~mL}(\mathrm{NTP}) / \mathrm{min}+5.0 \% \mathrm{O}_{2}$ in helium flowing at $16.0 \mathrm{~mL}(\mathrm{NTP}) / \mathrm{min}$; the pressure was atmospheric. The reactor was heated from room temperature at a rate of $2{ }^{\circ} \mathrm{C} / \mathrm{min}$ with the gases flowing and then held at $300{ }^{\circ} \mathrm{C}$ for 20 min before the reactor was cooled down.

In a separate flow reactor system, near-steady-state conversion data were obtained under the conditions stated in the preceding paragraph, except that the feed gas was a mixture of 5.0\% $\mathrm{CO}$ in helium flowing at 5.0,6.0, or $8.0 \mathrm{~mL}(\mathrm{NTP}) / \mathrm{min}+5.0 \% \mathrm{O}_{2}$ in helium flowing at 20.0, 24.0, or $32.0 \mathrm{~mL}(\mathrm{NTP}) / \mathrm{min}$. Catalytic reaction rates were calculated from low (differential) conversions $(<5 \%)$ determined at temperatures in the range of $180-200{ }^{\circ} \mathrm{C}$. In experiments determining the dependence of conversion on time on stream, the samples were kept on stream for up to $48 \mathrm{~h}$ to demonstrate stability at $210{ }^{\circ} \mathrm{C}$; the feed was $5.0 \% \mathrm{CO}$ in helium flowing at $4.0 \mathrm{~mL}(\mathrm{NTP}) / \mathrm{min}$ and $5.0 \% \mathrm{O}_{2}$ in helium flowing at $16.0 \mathrm{~mL}(\mathrm{NTP}) / \mathrm{min}$.

Scanning Transmission Electron Microscopy. STEM imaging experiments were performed on a JEOL 200CF (NEOARM) transmission electron microscope with an acceleration voltage of 200 $\mathrm{kV}$. The specimens were prepared by a direct dispersion of powder samples on lacey carbon grids. The HAADF images were acquired with a convergence angle of $28.5 \mathrm{mrad}$ and an inner collection angle of $55 \mathrm{mrad}$. 
Transmission Electron Microscopy. TEM images of as-prepared sample powders loaded onto copper grids were recorded with a Hitachi H-7700 microscope at an acceleration voltage of 100 $\mathrm{kV}$.

\section{X-ray Diffraction Crystallography}

XRD patterns of the samples were collected on a Philips X'Pert Pro Super diffractometer with a monochromatized $\mathrm{Cu} \mathrm{K} \alpha$ radiation source and a wavelength of $0.1542 \mathrm{~nm}$.

\section{Inductively Coupled Plasma Mass Spectrometry.}

The Pt loadings in the catalysts were determined by inductively coupled plasma mass spectrometry with a Thermo Scientific XSERIES 2 instrument.

\section{DFT Calculations.}

Periodic DFT calculations were performed using the projector augmented wave method as implemented in the Vienna ab initio simulation package (VASP). ${ }^{22}$ Energies were calculated using a $500 \mathrm{eV}$ plane-wave cutoff with a $2 \times 1 \times 1$ Monkhorst-Pack k-point grid. A range of generalized gradient approximation functionals (PBE, RPBE, and PBESol) were used. Electronic energies were converged to $10^{-6} \mathrm{eV}$, and all structures were relaxed until the forces were less than 0.05 $\mathrm{eV} / \AA$. The finite displacement method $(0.02 \AA)$ was used to calculate the entropic and zero-point energy corrections. For CO oxidation mechanism calculations, a $400 \mathrm{eV}$ plane-wave cutoff was used with the RPBE functional, and dispersion corrections were considered with the DFT-D3 method with Becke-Johnson damping. Barriers were calculated using the climbing image nudged elastic band and dimer methods.

\section{Conventional XAS Data Analysis.}

The EXAFS and XANES data were analyzed with the Demeter package. ${ }^{19}$ Pre-processing of data included alignment, edge calibration, deglitching, normalization, background subtraction, and 
conversion of data into a chi file for data fitting, performed with Athena. The energy at the Pt $\mathrm{L}_{3}$ edge, determined by the first inflection point of the absorption edge data characterizing the reference Pt foil, was calibrated to the reported energy, $11564.0 \mathrm{eV}$. EXAFS data were modeled with the Artemis package in Demeter. The continuous Cauchy wavelet transform (CCWT) was performed on the extracted EXAFS to visualize the data and assess how many scattering paths would be needed to fit the data. ${ }^{21}$ Larch $^{26}$ was used to perform the CCWT on EXAFS spectra at the $\mathrm{Pt}_{3}$ edge characterizing $\mathrm{Pt} / \mathrm{MgO}$ samples and $\mathrm{Pt}$ foil.

\section{Automated DFT-based Fitting of EXAFS Data.}

The EXAFS data were analyzed with the open-source X-ray Larch package. ${ }^{26}$ Data processing, such as alignment, edge calibration, deglitching, normalization, and background subtraction, was performed using the Python interface to Larch. The DFT-optimized structures were used to generate a feff.inp file using an in-house code. These feff.inp files were run in Larch, with FEFF used for generating scattering paths. The code uses Matplotlib for plotting the fitting results.

FEFF and DOS Modeling. All HERFD simulations used DFT-optimized (PBE-D3) structures and were performed with FEFF 9 using SCF and FMS cutoff radii of 6 and $9 \AA$, respectively, which ensured convergence of the spectra. ${ }^{34,35}$ The representative atomic potentials were chosen to reproduce chemical distinguishability for each atom type. On the basis of our experience with Pt-containing samples, we chose not to use a core-hole in the calculations. ${ }^{39,40}$ To properly simulate HERFD rather than the default XANES in FEFF, we also removed $1.8 \mathrm{eV}$ from the default corehole lifetime broadening of $5.2 \mathrm{eV}$. The self-energy was modeled using the density-dependent MPSE (many-pole self-energy) dielectric function approach based on a weighted average of the atomic loss functions. ${ }^{41}$ Moreover, to provide converged results up to about $200 \mathrm{eV}$ above the edge, the maximum angular momenta for the site basis set were raised to 5, 4, and 4 for $\mathrm{Pt}, \mathrm{O}$, and 
$\mathrm{Mg}$, respectively. Further, vibrational disorder was added using single-scattering Debye-Waller factors based on a correlated Debye model. The Debye temperature for this model was estimated for each system using average force constants for the first-shell Pt-O bonds obtained from the DFT structural simulations ${ }^{28,29}$.

\section{ASSOCIATED CONTENT}

Supplementary_Information_EXAFS_Fits

Supplementary_Information_EXAFS_Paths

Supplementary_Information_EXAFS_Fit_Parameters

\section{AUTHOR CONTRIBUTIONS}

Y.C. developed the catalyst synthesis, did IR characterization and catalytic reaction measurements with supervision by B.C.G., C.X.K. and A.R.K.; S.C., T.B. and Z.H. performed STEM measurements and analyzed data with supervision by M.C.; T.S. and R.R. performed the DFT calculations supervised by A.R.K.; Y.C., C.-Y.F., and A.S.H. performed the XAS experiments with supervision by S.R.B.; A.S.H., J.H. and R.R. performed the XAFS analysis; C.S and C.W. made the inductively coupled plasma mass spectrometry and x-ray diffraction crystallography measurements supervised by J.Z.; F.D.V. performed the HERFD-XANES data analysis. All authors analyzed and interpreted the results and contributed to the preparation of the manuscript. B.C.G. and A.R.K. conceived the project and oversaw all portions. Y.C., R.R., and T.S. contributed equally to this work.

\section{ACKNOWLEDGEMENTS}


Y.C. and B.C.G. were supported by the U.S. Department of Energy (DOE), Office of Science, Basic Energy Sciences (BES) grant DE-FG02-04ER15513. Stanford Synchrotron Radiation Lightsource (SSRL) of SLAC National Accelerator Laboratory is supported by BES under Contract No. DE-AC02-76SF00515. Co-ACCESS is supported by the BES Chemical Sciences, Geosciences, and Biosciences Division. C.X.K. and A.R.K. were supported by the U.S. DOE, Office of Science, BES grant DE-SC0020320. Y.C. gratefully acknowledge the China Scholarship Council (CSC. No. 201806340062) for financial support. R.R. acknowledges support from U.S. DOE (DE-SC0020320, for DFT calculations) and SLAC National Accelerator Laboratory (for EXAFS analysis). T.S. was supported by start-up funds provided by UC Davis College of Engineering. Z. H. was supported by the U.S. Office of Basic Energy Sciences, Chemical Sciences, Geosciences, and Biosciences Division, Catalysis Science Program. Electron microscopy was performed at Oak Ridge National Laboratory's (ORNL) Center for Nanophase Materials Sciences (CNMS), which is a DOE Office of Science User Facility (T. B., S. C. M. C.). The DFT calculations in this work were performed using the resources of the National Energy Research Scientific Computing Center (NERSC), a U.S. DOE Office of Science User Facility located at Lawrence Berkeley National Laboratory, operated under Contract No. DE-AC0205CH11231. Nudged elastic band calculations reported in this work used the Extreme Science and Engineering Discovery Environment (XSEDE), supported by National Science Foundation grant number ACI-1548562.

\section{ADDITIONAL INFORMATION}

Supporting information is available in the online version of the paper. Correspondence and requests for materials should be addressed to C.X.K, S.R.B., B.C.G., or A.R.K. The ASE database 
file and the EXAFS fitting routines are available on GitHub at https://github.com/kul-group/kulrepository/tree/main/repo_2021_JACS_Theory-Guided and https://github.com/kulgroup/QuantEXAFS, respectively.

\section{NOTES}

The authors declare no conflict of interest.

\section{REFERENCES}

1. Resasco, J.; DeRita, L.; Dai, S.; Chada, J. P.; Xu, M.; Yan, X.; Finzel, J.; Hanukovich, S.; Hoffman, A. S.; Graham, G. W.; Bare, S. R.; Pan, X.; Christopher, P. Uniformity Is Key in Defining Structure-Function Relationships for Atomically Dispersed Metal Catalysts: The Case of $\mathrm{Pt} / \mathrm{CeO}_{2}$. J. Am. Chem. Soc. 2020, 142, 169-184.

2. DeRita, L.; Resasco, J.; Dai, S.; Boubnov, A.; Thang, H. V.; Hoffman, A. S.; Ro, I.; Graham, G. W.; Bare, S. R.; Pacchioni, G.; Pan, X.; Christopher, P. Structural Evolution of Atomically Dispersed Pt Catalysts Dictates Reactivity. Nat. Mater. 2019, 18 (7), 746-751.

3. Zhu, Y.; Yuk, S. F.; Zheng, J.; Nguyen, M.-T.; Lee, M.-S.; Szanyi, J.; Kovarik, L.; Zhu, Z.; Balasubramanian, M.; Glezakou, V.-A.; Fulton, J. L.; Lercher, J. A.; Rousseau, R.; Gutiérrez, O. Y. Environment of Metal-O-Fe Bonds Enabling High Activity in $\mathrm{CO}_{2}$ Reduction on Single Metal Atoms and on Supported Nanoparticles. J. Am. Chem. Soc. 2021, 143, 5540-5549.

4. Zhang, Z.; Zhu, Y.; Asakura, H.; Zhang, B.; Zhang, J.; Zhou, M.; Han, Y.; Tanaka, T.; Wang, A.; Zhang, T.; Yan, N. Thermally Stable Single Atom Pt/M- $\mathrm{Al}_{2} \mathrm{O}_{3}$ for Selective Hydrogenation and CO Oxidation. Nat. Commun. 2017, 8, 16100. 
5. Hoffman, A. S.; Debefve, L. M.; Zhang, S. J.; Perez-Aguilar, J. E.; Conley, E. T.; Justl, K. R.; Arslan, I.; Dixon, D. A.; Gates, B. C. Beating Heterogeneity of Single-Site Catalysts: MgOSupported Iridium Complexes. ACS Catal. 2018, 8, 3489-3498.

6. Kwon, Y.; Kim, T. Y.; Kwon, G.; Yi, J.; Lee, H. Selective Activation of Methane on SingleAtom Catalyst of Rhodium Dispersed on Zirconia for Direct Conversion. J. Am. Chem. Soc. 2017, 139, 17694-17699.

7. Li, L.; Chang, X.; Lin, X.; Zhao, Z.-J.; Gong, J. Theoretical Insights into Single-Atom Catalysts. Chem. Soc. Rev. 2020, 49 (22), 8156-8178.

8. Mitchell, S.; Pérez-Ramírez, J. Single Atom Catalysis: A Decade of Stunning Progress and the Promise for a Bright Future. Nat. Commun. 2020, 11, 4302.

9. Chen, Y. Z.; Sun, H. L.; Gates, B. C. Prototype Atomically Dispersed Supported Metal Catalysts: Iridium and Platinum. Small, 2020, 17, 2004665.

10. Flytzani-Stephanopoulos, M.; Gates, B. C. Atomically dispersed supported metal catalysts. Annu. Rev. Chem. Biomol. Eng. 2012, 3, 545-574.

11. Samantaray, M. K.; D’Elia, V.; Pump, E.; Falivene, L.; Harb, M.; Chikh, S. O.; Cavallo, L.; Basset, J.-M. The Comparison between Single Atom Catalysis and Surface Organometallic Catalysis. Chem. Rev. 2020, 120, 734-813.

12. Qin, R.; Liu, K.; Wu, Q.; Zheng, N. Surface Coordination Chemistry of Atomically Dispersed Metal Catalysts. Chem. Rev. 2020, 120, 11810-11899.

13. Gates, B. C. Atomically Dispersed Supported Metal Catalysts: Seeing Is Believing. Trends Chem. 2019, 1, 99-110.

14. Kaiser, S. K.; Chen, Z.; Faust Akl, D.; Mitchell, S.; PerezRamirez, J. Single-Atom Catalysts across the Periodic Table. Chem. Rev. 2020, 120 (21), 11703-11809. 
15. Lu, Y.; Wang, J.; Yu, L.; Kovarik, L.; Zhang, X.; Hoffman, A. S.; Gallo, A.; Bare, S. R.; Sokaras, D.; Kroll, T.; Dagle, V.; Xin, H.; Karim, A. M. Identification of the active complex for $\mathrm{CO}$ oxidation over single-atom Ir-on- $\mathrm{MgAl}_{2} \mathrm{O}_{4}$ catalysts. Nat. Catal. 2019, 2 (2), 149- 156.

16. Maurer, F.; Jelic, J.; Wang, J.; Gänzler, A.; Dolcet, P.; Wöll, C.; Wang, Y.; Studt, F.; Casapu, M.; Grunwaldt, J.-D. Tracking the formation, fate and consequence for catalytic activity of Pt single sites on $\mathrm{CeO}_{2}$. Nat. Catal. 2020, 3, 824-833.

17. T Timoshenko, J.; Frenkel, A. I. “Inverting” X-ray Absorption Spectra of Catalysts by Machine Learning in Search for Activity Descriptors. ACS Catal. 2019, 9, 10192-10211.

18. Pennycook, S. J.; Jesson, D. E. High-resolution Z-contrast imaging of crystals. Ultramicroscopy 1991, 37 (1-4), 14-38.

19. Ravel, B.; Newville, M. ATHENA, ARTEMIS, HEPHAESTUS: data analysis for X-ray absorption spectroscopy using IFEFFIT. J. Synchrotron Radiat. 2005, 12, 537-541.

20. Liu, L.; Lopez-Haro, M.; Lopes, C. W.; Li, C.; Concepcion, P.; Simonelli, L.; Calvino, J. J.; Corma, A. Regioselective Generation and Reactivity Control of Subnanometric Platinum Clusters in Zeolites for High-Temperature Catalysis. Nat. Mater. 2019, 18, 866-873.

21. Muñoz, M.; Argoul, P.; Farges, F. Continuous Cauchy wavelet transform analyses of EXAFS spectra: A qualitative approach. Am. Mineral. 2003, 88, 694-700.

22. Kresse, G.; Hafner, J. Ab Initio Molecular Dynamics for Liquid Metals. Phys. Rev. B: Condens. Matter Mater. Phys. 1993, 47, 558-561.

23. Prada, S.; Giordano, L.; Pacchioni, G. Charging of Gold Atoms on Doped MgO and CaO: Identifying the Key Parameters by DFT Calculations. J. Phys. Chem. C 2013, 117, 9943-9951. 
24. Lym, J.; Wittreich, G. R.; Vlachos, D. G. A Python Multiscale Thermochemistry Toolbox (PMuTT) for Thermochemical and Kinetic Parameter Estimation. Comput. Phys. Commun. 2020, $247,106864$.

25. Larsen, A. H.; Mortensen, J. J.; Blomqvist, J.; Castelli, I. E.; Christensen, R.; Dulak, M.; Friis, J.; Groves, M. N.; Hammer, B.; Hargus, C.; Hermes, E. D.; Jennings, P. C.; Jensen, P. B.; Kermode, J.; Kitchin, J. R.; Kolsbjerg, E. L.; Kubal, J.; Kaasbjerg, K.; Lysgaard, S.; Maronsson, J. B.; Maxson, T.; Olsen, T.; Pastewka, L.; Peterson, A.; Rostgaard, C.; Schiotz, J.; Schutt, O.; Strange, M.; Thygesen, K. S.; Vegge, T.; Vilhelmsen, L.; Walter, M.; Zeng, Z.; Jacobsen, K. W. The atomic simulation environment - A Python library for working with atoms. J. Phys.: Condens. Matter 2017, 29, 273002.

26. Newville, M. Larch: An Analysis Package for XAFS and Related Spectroscopies. J. Phys.: Conf. Ser. 2013, 430, 012007.

27. Sarma, B. B.; Plessow, P. N.; Agostini, G.; Concepcion, P.; Pfander, N.; Kang, L.; Wang, F. R.; Studt, F.; Prieto, G. Metal-specific" reactivity in single-atom catalysts: CO oxidation on 4d and 5d transition metals atomically dispersed on MgO. J. Am. Chem. Soc. 2020, 142 (35), $14890-14902$.

28. Vila, F. D.; Rehr, J. J.; Rossner, H. H.; Krappe, H. J. Theoretical x-ray absorption DebyeWaller factors. Phys. Rev. B: Condens. Matter Mater. Phys. 2007, 76, 014301.

29. Vila, F. D.; Hayashi, S. T.; Rehr, J. J. Efficient calculation of the negative thermal expansion in $\mathrm{ZrW}_{2} \mathrm{O}_{8}$. Front. Chem. 2018, 6, 296.

30. Penner-Hahn, J. E. X-ray absorption spectroscopy, Elsevier Ltd.: New York, 2003. 
31. Mistry, H.; Behafarid, F.; Bare, S. R.; Roldan Cuenya, B. Pressure-dependent effect of hydrogen adsorption on structural and electronic properties of $\mathrm{Pt} / \gamma-\mathrm{Al}_{2} \mathrm{O}_{3}$ nanoparticles. ChemCatChem 2014, 6, 348-352.

32. Stavale, F.; Shao, X.; Nilius, N.; Freund, H. J.; Prada, S.; Giordano, L.; Pacchioni, G. Donor characteristics of transition-metaldoped oxides: $\mathrm{Cr}$-doped $\mathrm{MgO}$ versus Mo-doped CaO. J. Am. Chem.Soc. 2012, 134, 11380-11383.

33. Hoffman, A. S.; Sokaras, D.; Zhang, S. J.; Debefve, L. M.; Fang, C. Y.; Gallo, A.; Kroll, T.; Dixon, D. A.; Bare, S. R.; Gates, B. C. High-Energy-Resolution X-ray Absorption Spectroscopy for Identification of Reactive Surface Species on Supported Single-Site Iridium Catalysts. Chem. - Eur. J. 2017, 23, 14760-14768.

34. Rehr, J. J.; Kas, J. J.; Prange, M. P.; Sorini, A. P.; Takimoto, Y.; Vila, F. Ab Initio Theory and Calculations of X-ray Spectra. C. R. Phys. 2009, 10, 548-559.

35. Rehr, J. J.; Kas, J. J.; Vila, F. D.; Prange, M. P.; Jorissen, K. Parameter-free calculations of Xray spectra with FEFF9. Phys. Chem. Chem. Phys. 2010, 12, 5503-5513.

36. Hellman, A.; Klacar, S.; Groenbeck, H. Low temperature CO oxidation over supported ultrathin MgO films. J. Am. Chem. Soc. 2009, 131, 16636.

37. Babucci, M.; Guntida, A.; Gates, B. C. Atomically Dispersed Metals on Well-Defined Supports including Zeolites and Metal Organic Frameworks: Structure, Bonding, Reactivity, and Catalysis. Chem. Rev. 2020, 120, 11956-11985.

38. Hoffman, A. S.; Singh, J. A.; Bent, S. F.; Bare, S. R. In situ observation of phase changes of a silica-supported cobalt catalyst for the Fischer-Tropsch process by the development of a synchrotroncompatible in situ/operando powder X-ray diffraction cell. J. Synchrotron Radiat. 2018, 25 (6), 1673-1682. 
39. Vila, F. D.; Rehr, J. J.; Kelly, S. D.; Bares, S. R. Operando Effects on the Structure and Dynamics of $\mathrm{Pt}_{\mathrm{n}} \mathrm{Sn}_{\mathrm{m}} / \gamma-\mathrm{Al}_{2} \mathrm{O}_{3}$ from $\mathrm{Ab}$ Initio Molecular Dynamics and X-ray Absorption Spectra. J. Phys. Chem. C 2013, 117, 12446-12457.

40. Vila, F.; Rehr, J.; Kas, J.; Nuzzo, R.; Frenkel, A. Dynamic structure in supported Pt nanoclusters: real-time density functional theory and X-ray spectroscopy simulations. Phys. Rev. B: Condens. Matter Mater. Phys. 2008, 78, 121404.

41. Kas, J. J.; Vinson, J.; Trcera, N.; Cabaret, D.; Shirley, E. L.; Rehr, J. J. Many-pole model of inelastic losses applied to calculations of XANES. J. Phys. Conf. Ser. 2009, 190, 012009. 